DOI: $10.24850 / \mathrm{j}$-tyca-2021-04-08

Artículos

\title{
Tratamiento del diclofenaco en solución acuosa mediante electro-oxidación utilizando electrodos de DDB
}

\section{Treatment of diclofenac in aqueous solution by electro- oxidation using BDD electrodes}

Evelyn A. Hernández ${ }^{1}$ ORCID: https://orcid.org/0000-0003-0785-9558

Monserrat Castañeda² ORCID: https://orcid.org/0000-0002-4585-1285

Ivonne Linares33, ORCID: https://orcid.org/0000-0001-7302-8491

Perla T. Almazán ${ }^{4}$ ORCID: https://orcid.org/0000-0002-7136-1212

Guadalupe Vázquez ${ }^{5}$ ORCID: https://orcid.org/0000-0002-7718-769X

Verónica Martínez 6 ORCID: https://orcid.org/0000-0003-4977-9249

${ }^{1}$ Instituto Interamericano de Tecnología y Ciencias del Agua (IITCA), Universidad Autónoma del Estado de México, Toluca, Estado de México, México, ehernandezr224@alumno.uaemex.mx 
2Instituto Interamericano de Tecnología y Ciencias del Agua (IITCA), Universidad Autónoma del Estado de México, Toluca, Estado de México, México, mcastanedaj042@alumno.uaemex.mx

3Instituto Interamericano de Tecnología y Ciencias del Agua (IITCA), Universidad Autónoma del Estado de México, Toluca, Estado de México, México, ilinaresh@uaemex.mx

${ }^{4}$ Conacyt-Instituto Nacional de Investigaciones Nucleares, Departamento de Química, Ocoyoacac, Estado de México, México, perla.almazan@conacyt.mx

5Instituto Interamericano de Tecnología y Ciencias del Agua (IITCA), Universidad Autónoma del Estado de México, Toluca, Estado de México, México, gvazquezm@uaemex.mx

6Instituto Interamericano de Tecnología y Ciencias del Agua (IITCA), Universidad Autónoma del Estado de México, Toluca, Estado de México, México,mmirandav@uaemex.mx

Autora para correspondencia: Ivonne Linares, ilinaresh@uaemex.mx

\section{Resumen}

El consumo de productos farmacéuticos como los antiinflamatorios no esteroideos, entre ellos el ácido 2-[2-(2,6-dicloroanilino)fenil]acético, mejor conocido como diclofenaco, se ha incrementado debido a su fácil adquisición; como consecuencia existen repercusiones al ambiente, pues es excretado a través de la orina, ocasionando un riesgo potencial al ser 
descargados en cuerpos de agua, y creando persistencia en el ambiente y en los procesos de tratamiento de aguas residuales. Por tal razón es necesario utilizar procesos alternativos de oxidación avanzada, debido a que han demostrado ser eficientes en la degradación de compuestos xenobióticos. El objetivo de esta investigación fue aplicar un sistema de electro-oxidación y fotoelectro-oxidación utilizando electrodos de diamante dopados con boro (DDB) mediante una configuración DDB-DDB, con la finalidad de determinar las variables de operación para la oxidación de diclofenaco en solución acuosa. Las condiciones óptimas del estudio fueron las siguientes: intensidad de corriente de 1 A (densidad de corriente $\left.(\mathrm{J})=25.29 \mathrm{~A} / \mathrm{m}^{2}\right)$; el valor de $\mathrm{pH}$ de la muestra $(5-6) ; \mathrm{NaCl}$ como electrolito soporte, en ausencia de luz UV, y tiempo de tratamiento de 360 minutos. La eficiencia de remoción de diclofenaco fue $97.8 \%$, el carbono orgánico total (COT) y la demanda química de oxígeno (DQO) presentaron eficiencias del $64.4 \%$ y $89.3 \%$, respectivamente, mientras que la demanda bioquímica de oxígeno $\left(\mathrm{DBO}_{5}\right)$ disminuyó después del tratamiento. La cinética de degradación del diclofenaco se ajustó a un modelo de pseudo primer orden, con un tiempo de vida media de 63.89 minutos y una $\mathrm{k}=1.08 \times 10^{-2} \mathrm{~min}^{-1}$. Los porcentajes de remoción de diclofenaco empleando cromatografía de líquidos (HPLC) fueron $99.64 \%$, $98.76 \%$ y $95.15 \%$ para las concentraciones de 150, 50 y 10 mg/l, respectivamente.

Palabras clave: oxidación electroquímica, diclofenaco, luz UV, ánodo de DDB, POA. 
2021, Instituto Mexicano de Tecnología del Agua

Open Access bajo la licencia CC BY-NC-SA 4.0

(https://creativecommons.org/licenses/by-nc-sa/4.0/)

\section{Abstract}

The consumption of pharmaceutical products such as non-steroidal antiinflammatories, including 2- [2- (2,6-dichloroanilino) phenyl] acetic acid, better known as diclofenac, has been increasing due to its easy acquisition, as a consequence the environment has had repercussions since it is excreted through the urine, causing a potential risk to be discharged into water bodies and creating persistence in the environment and in the processes of wastewater treatment. Therefore, it is necessary to use alternative processes of advanced oxidation because they have proven to be efficient in the degradation of xenobiotic compounds. The objective of this research was to apply an electro-oxidation system and photoelectro-oxidation by boron-doped diamond (BDD) electrodes using a BDD-BDD configuration to determine the operation variables for the oxidation of diclofenac in aqueous solution. The optimal conditions of the study were: current intensity of $1 \mathrm{~A}$ (current density $(\mathrm{J})=25.29 \mathrm{~A} / \mathrm{m}^{2}$ ), the $\mathrm{pH}$ of the sample (5-6); $\mathrm{NaCl}$ as supporting electrolyte, in the absence of UV light, and treatment time of 360 minutes. The removal efficiency of diclofenac was $97.8 \%$, the TOC, COD showed efficiencies of $64.4 \%$ and $89.3 \%$, respectively, whereas the biochemical oxygen demand (BOD5) decrease after of treatment. The degradation kinetic of diclofenac was adjusted to a pseudo first order model, with a half-life time of 63.89 minutes and $a \mathrm{k}=1.08 \times 10^{-2} \mathrm{~min}^{-1}$. The removal percentages of diclofenac using liquid chromatography (HPLC) were $99.64 \%, 98.76 \%$ and $95.15 \%$ for the concentrations of 150,50 and $10 \mathrm{mg} / \mathrm{l}$, respectively. 
Keywords: Electrochemical oxidation, diclofenac, UV light, BDD anode, AOP.

Recibido: 30/01/2019

Aceptado: $11 / 09 / 2020$

\section{Introducción}

El uso de antinflamatorios no esteroideos como el ácido 2-[2-(2,6 dicloroanilino)fenil]acético, conocido comúnmente como diclofenaco (DCF), se ha incrementado en los últimos años en el mundo. En México, este fármaco se encuentra en el cuadro básico del sector salud, y es utilizado para aliviar dolor e inflamación, así como dolores asociados con afecciones reumáticas (McGettigan \& Henry, 2013). El diclofenaco comercial es la sal de un ácido débil con coeficiente de partición de 13.4 y pKa de 4.15, tiene una baja solubilidad y débil capacidad de ionización; sin embargo, la presencia de átomos de nitrógeno, oxígeno, cloro y sodio promueven polaridad en la molécula, propiciando su solubilidad en el agua (Žilnik, Jazbinšek, Hvala, Vrečer, \& Klamtc, 2007); además, presenta propiedades ácido-base, ya que actúa como aceptor o donador de 
protones por la presencia del grupo amino (NH) (Žilnik et al., 2007). Es un compuesto emergente, pues su presencia en medio acuoso ocasiona daños a la salud humana y al ambiente en bajas concentraciones (Liu \& Wong, 2013; Archer, Petrie, Kasprzyk-Hordernb, \& Wolfaardtac, 2017), además de que los sistemas de tratamiento convencionales son poco eficientes para removerlos del agua. De acuerdo con el Consejo Consultivo del Agua, hasta el año 2015 un 70 \% de los cuerpos de agua en México estaba contaminado, al recibir descargas de aguas residuales sin tratamiento, ya fueran de tipo municipal o industrial. Dentro de los contaminantes detectados se encuentran los compuestos emergentes, los cuales no están regulados y tienen actividad tóxica o mutagénica (Barceló, \& López-de-Alda, 2008). Las concentraciones de compuestos farmacéuticos en aguas residuales urbanas e incluso en agua potable han sido reportadas en escala de $\mu \mathrm{g} / \mathrm{l}$ y $\mathrm{mg} / \mathrm{l}$; su toxicidad en organismos acuáticos se ha evaluado en diversos estudios, los cuales muestran efectos citotóxicos en células hepáticas y renales incluso a bajas concentraciones (Bolong, Ismail, Salim, \& Matsuura, 2009; Daughton \& Brooks, 2011), promoviendo que se implementen nuevas tecnologías para su degradación y eliminación debido a que por su mineralización incompleta pueden conducir a la formación de sustancias químicas tóxicas (Rodríguez, Márquez, León, \& Alvarez, 2013). Se han aplicado diferentes tecnologías para la remoción y/o degradación del diclofenaco: tratamientos biológicos (Fatehifar, Borghei, \& Ekhlasi-Nia, 2018; Falås, Wick, Joss, \& Ternes, 2016); fisicoquímicos (Lonappan, Rouissi, Yuxue, Kaur-Brar, \& Surampalli, 2019); y tratamientos terciarios, como la hidrodecloración catalítica (Nieto-Sandoval, Munoz, De-Pedro, \& Casas, 
2018) y la irradiación con electrones (Tominaga, Dos-Santos-Batista, Silva-Costa-Teixeira, \& Borrely, 2018); la desventaja de estas tecnologías es que forman subproductos, y en el caso de los procesos biológicos es necesario la adaptación de los microrganismos y aumentar el tiempo de retención (Vieno \& Sillanpää, 2014). Dentro de los procesos fisicoquímicos se han aplicado precipitación química, filtración por membrana, adsorción y desinfección; no obstante, los porcentajes de remoción son bajos y la desventaja es que el contaminante sólo cambia de fase (Bolong et al., 2009; De-Franco, De-Carvalho, Bonetto, De-Pelegrini-Soares, \& Féris, 2018). Los tratamientos más satisfactorios para la remoción de compuestos persistentes son los procesos de oxidación avanzada (POA), capaces de producir cambios sustanciales en la estructura química de los contaminantes, por la generación y el uso de especies transitorias con un alto poder oxidante, como el radical hidroxilo $(\cdot \mathrm{OH})$ (Pourzamani, Hajizadeh and \& Mengelizadeh, 2018). Entre los POA más utilizados para la degradación del diclofenaco están la fotocatálisis heterogénea (Casillas et al., 2017; Lu et al., 2017; Martínez, Canle, Fernández, Santaballa, \& Faria, 2011), foto-Fenton y variantes Fenton (Pérez-Estrada et al., 2005; Araujo et al., 2011; Bae, Kim, \& Lee, 2013; Venegas, 2013; Lu et al., 2017). Dentro de los POA se encuentra la oxidación electroquímica o electro-oxidación (EO) utilizada para el tratamiento de aguas residuales con contaminantes orgánicos persistentes (Cao, Wang, \& Zhao, 2017; Tominaga et al., 2018). La EO degrada los contaminantes hasta su mineralización total. En este proceso, la capacidad de oxidación del ánodo utilizado depende de su naturaleza junto con variables operacionales como $\mathrm{pH}$, concentración del contaminante, temperatura y agitación. E 
proceso de electro-oxidación se realiza en una celda electroquímica, compuesta por un electrodo de trabajo (ánodo) y un contraelectrodo que completa el circuito (cátodo), los cuales se sumergen en una solución (electrolito) capaz de permitir la transferencia de electrones; si esto no ocurre, se agregan iones conocidos como "electrolito soporte" (Barrera, 2014). Los electrodos de diamante dopados con boro (DDB) poseen una alta estabilidad anódica, amplia ventana de potencial, alta conductividad; son inertes y son electrodos no activos, pues tienen débiles propiedades de adsorción; promueven la oxidación de compuestos orgánicos por vía radicalaria, a partir de la formación de radicales hidroxilo (García-Montoya et al., 2015).

Por otro lado, en la fotoelectro-oxidación (FEO), la presencia de radiación UV provoca un efecto sinérgico que promueve una alta tasa de oxidación debido a una mayor generación de radicales $\cdot \mathrm{OH}$ en comparación con los procesos individuales, obteniendo así mejores eficiencias. Con base en lo anterior, en el presente trabajo de investigación se propuso un proceso de electro-oxidación y fotoelectrooxidación para el tratamiento de soluciones acuosas de diclofenaco $\left(\mathrm{C}_{0}=\right.$ 150,50 y $10 \mathrm{mg} / \mathrm{l}$ ) utilizando una configuración electródica DDB-DDB. El proceso se realizó durante $6 \mathrm{~h}$ a temperatura ambiente, $\mathrm{pH}$ (5-6), estudiando el efecto de la intensidad de corriente $0.5 \mathrm{~A}\left(\mathrm{~J}=12.64 \mathrm{~A} / \mathrm{m}^{2}\right)$ y $1.0 \mathrm{~A}\left(\mathrm{~J}=25.29 \mathrm{~A} / \mathrm{m}^{2}\right)$, del electrolito soporte $\left(\mathrm{NaCl}\right.$ y $\left.\mathrm{Na}_{2} \mathrm{SO}_{4}\right)$, y la presencia o ausencia de radicación UV $(\Lambda=254 \mathrm{~nm})$. Este sistema permitió incrementar el potencial de oxidación, haciendo uso de la reducción electroquímica del oxígeno para la generación de peróxido de hidrógeno. 
Tecnología y

Ciencias $\stackrel{\Xi}{\approx}$ gua
2021, Instituto Mexicano de Tecnología del Agua

Open Access bajo la licencia CC BY-NC-SA 4.0

(https://creativecommons.org/licenses/by-nc-sa/4.0/)

\title{
Metodología
}

\section{Preparación de las soluciones acuosas}

Se prepararon soluciones acuosas empleando la sal de diclofenaco (DCF) de sodio (Sigma Aldrich México, CAS: 15307-79-6) en agua desionizada. Las concentraciones iniciales de las soluciones fueron 150, 50 y $10 \mathrm{mg} / \mathrm{l}$.

\section{Métodos de caracterización}

\author{
Espectroscopia UV-Vis
}


Se realizó el barrido de la solución acuosa de DFC a $150 \mathrm{mg} / \mathrm{l}$ en un espectrofotómetro UV-Vis Cary Varian, de 400 a $200 \mathrm{~nm}$ para determinar la absorbancia máxima ( $\lambda_{\text {máx }}$ ). El $\lambda_{\text {máx }}$ se observó a $276 \mathrm{~nm}$, la cual es similar a la reportada por Casillas-García, Tzompantzi-Morales, CarbajalArizaga, López-Ganoa y Barrera-Rodríguez (2017) (275 nm). Posteriormente se procedió a realizar una curva de calibración para analizar el comportamiento del DCF durante los tratamientos.

\section{Espectroscopia infrarroja}

Las soluciones acuosas se caracterizaron por espectroscopía de infrarrojo utilizando el equipo Shimadzu Fourier Affinity15, con un rango de longitud de onda de 4 000-400 $\mathrm{cm}^{-1}$ y con 45 barridos utilizando una celda ATR para identificar los grupos funcionales característicos.

\section{Fluorescencia}


Todos los espectros de fluorescencia se efectuaron en un espectrofluorómetro (Modelo LS-55, Perkin Elmer, EUA) equipado con una lámpara de xenón de 150 watts como fuente de excitación. El espectrofluorómetro fue controlado con el software WINLAB (Perkin Elmer, EUA, 2000) operado desde una computadora de escritorio. Las matrices de excitación/emisión en 3D (EEM-3D, por sus siglas en inglés) se construyeron colectando 45 espectros de emisión con longitudes de emisión $\left(\lambda_{e m}\right)$ entre 250 y 600 nm, mientras que la longitud de excitación ( $\lambda_{\text {exc }}$ ) se varió entre 200 y $420 \mathrm{~nm}$ cada $5 \mathrm{~nm}$. Para evitar las perturbaciones Rayleigh y Raman del agua se utilizó un filtro a $290 \mathrm{~nm}$ y se sustrajo un blanco de agua desionizada.

\section{Análisis fisicoquímicos}

Las muestras se caracterizaron evaluando los siguientes parámetros: demanda química de oxígeno (DQO), demanda bioquímica de oxígeno $\left(\mathrm{DBO}_{5}\right)$ y carbono orgánico total (COT), la $\mathrm{DQO}$ y $\mathrm{DBO}_{5}$ se realizaron de acuerdo con lo establecido en las Normas Mexicanas NMX-AA-030/1SCFI-2012 y NMX-AA-028-SCFI-2001, respectivamente. El COT se determinó empleando el equipo Total Organic Carbon Analyzer O. I. Analytical 1020A. 


\section{Cromatografía de líquidos (HPLC)}

Las muestras del proceso con las mejores condiciones de oxidación se analizaron en un equipo Agilent 1260 Infinity LC con un detector de diodos (DAD); se utilizó una columna $\mathrm{C}_{18}$ Zorbax SB $(5 \mu \mathrm{m}, 150 \mathrm{~mm} \times 4.6 \mathrm{~mm})$. La fase móvil usada fue de ácido acético $0.2 \%$ (v/v) y acetonitrilo; el flujo fue de $1 \mathrm{ml} / \mathrm{min}$, la temperatura de columna de $30^{\circ} \mathrm{C}$ y el detector a $275 \mathrm{~nm}$.

\section{Tratamiento de electro-oxidación}

Se utilizó una celda electrolítica (Figura 1) asistida con electrodos de DDB de la marca Condias ${ }^{\circledR}$ ( 3 ánodos / 2 cátodos). Las dimensiones de cada electrodo de DDB fueron de $3.0 \times 20.5 \times 0.2 \mathrm{~cm}$, con una superficie de contacto real de $0.011 \mathrm{~m}^{2}\left(115.32 \mathrm{~cm}^{2}\right)$, la distancia entre los electrodos fue de $0.3 \mathrm{~cm}$ y la relación área/volumen fue de $28.83 \mathrm{~m}^{2} / \mathrm{m}^{3}$. Se trataron 0.4 I de la solución de DCF a $150 \mathrm{mg} / \mathrm{l}$ durante seis horas a temperatura ambiente, con agitación continua (400 rpm), y pH entre 5 y 6; se usó 
como electrolito soporte $\mathrm{NaCl}(2.5 \mathrm{~g} / \mathrm{l})$ y $\mathrm{Na}_{2} \mathrm{SO}_{4}(1.25 \mathrm{~g} / \mathrm{l})$. Se analizaron dos intensidades de corriente: $0.5 \mathrm{~A}\left(\mathrm{~J}=12.64 \mathrm{~A} / \mathrm{m}^{2}\right)$ y $1.0 \mathrm{~A}(\mathrm{~J}=25.29$ $\mathrm{A} / \mathrm{m}^{2}$ ) en presencia o ausencia de luz UV, utilizando una lámpara de onda corta $(\lambda=254 \mathrm{~nm})$. La corriente directa se suministró con una fuente de poder EXTECH 382213.

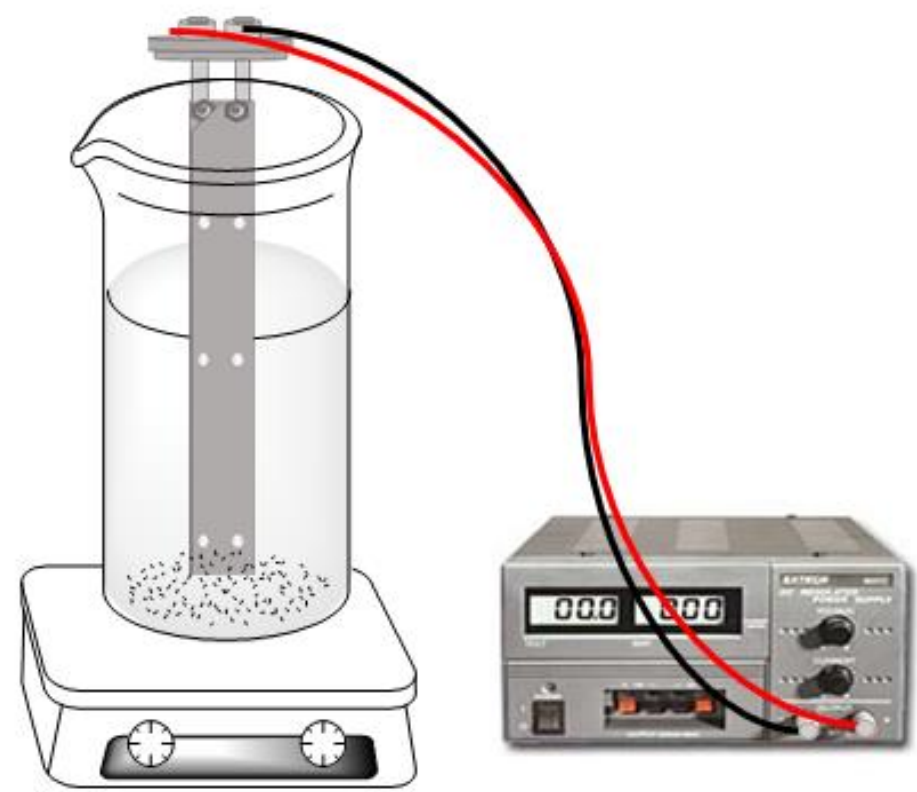

Figura 1. Celda electroquímica usada para el proceso de electrooxidación.

Los experimentos se llevaron a cabo con base en un diseño experimental $2^{3}$, y los resultados se analizaron mediante el Algoritmo de Yates y ANOVA (Material suplementario I y II), con el fin de obtener las mejores condiciones experimentales. Las variables de estudio fueron 
intensidad de corriente $\left(0.5\left(\mathrm{~J}=12.64 \mathrm{~A} / \mathrm{m}^{2}\right)\right.$ y $\left.1.0 \mathrm{~A}\left(\mathrm{~J}=25.29 \mathrm{~A} / \mathrm{m}^{2}\right)\right)$ electrolito soporte $\left(\mathrm{NaCl}\right.$ y $\left.\mathrm{Na}_{2} \mathrm{SO}_{4}\right)$ y presencia o ausencia de luz UV.

Una vez obtenidas las mejores condiciones evaluando una concentración de DFC, las soluciones acuosas de diclofenaco a 10 y 50 $\mathrm{mg} / \mathrm{l}$ se trataron a intensidad de corriente de $1 \mathrm{~A}\left(\mathrm{~J}=25.29 \mathrm{~A} / \mathrm{m}^{2}\right)$, en ausencia de luz UV y utilizando $\mathrm{NaCl}$ como electrolito soporte.

Los electrodos de DDB se limpiaron con una solución de $\mathrm{Na}_{2} \mathrm{SO}_{4}$ (5 $\mathrm{g} / \mathrm{L} ; \mathrm{pH}=2$ ), y se le suministró corriente eléctrica durante 20 minutos, a la intensidad de corriente de trabajo; posteriormente se enjuagaron con agua deionizada. Este proceso de limpieza se realizó al finalizar cada experimento de electro-oxidación.

Durante el proceso de electro-oxidación se tomaron alícuotas a diferentes tiempos para analizar la concentración de la DQO y de DCF por UV-Vis a 275 nm; y a las mejores condiciones de operación se determinó COT, fluorescencia, IR y la concentración de DCF por HPLC.

\section{Resultados}

\section{Caracterización de soluciones acuosas}




\section{Espectroscopia infrarroja}

Como parte de la caracterización inicial del DCF se obtuvo el espectro de IR del DCF sódico en forma sólida y acuosa (Figura 2), en los cuales se pudieron localizar las bandas características para ambas fases, entre las cuales se encuentran N-H alargamiento (3 385.07 y $3259.69 \mathrm{~cm}^{-1}$ (sólido) y $3354.16 \mathrm{~cm}^{-1}$ (solución acuosa)); C-H aromático (3 $076.46 \mathrm{~cm}^{-1}$ y 3 $069.76 \mathrm{~cm}^{-1}$ para el sólido y la solución acuosa, respectivamente); $C=C$ aromático (1 597.05 y $1589.40 \mathrm{~cm}^{-1}$ (sólido) y $1507.16 \mathrm{~cm}^{-1}$ (solución acuosa)); C-H alifático (1 460.11 y $1448.54 \mathrm{~cm}^{-1}$ (sólido) y $1414.78 \mathrm{~cm}$ 1 (solución acuosa)); Ar-N alargamiento (1 280.73, 1236.37 y 1232.51 $\mathrm{cm}^{-1}$ (sólido) y $1239.28 \mathrm{~cm}^{-1}$ (solución acuosa)); C=O grupo carboxílico (1 $286.52 \mathrm{~cm}^{-1}$ (sólido) y $1241.07 \mathrm{~cm}^{-1}$ (solución acuosa)); C-Cl (634.58 y $607.57 \mathrm{~cm}^{-1}$ (sólido), 631.69 y $635.55 \mathrm{~cm}^{-1}$ (solución acuosa)). Asimismo, se puede apreciar la señal característica de la sal en el espectro del compuesto sólido correspondiente a $\mathrm{COO}^{-} \mathrm{Na}^{+}$con un valor de 1 $354.93 \mathrm{~cm}^{-1}$ (Krajišnik et al., 2013; Bhadra, Seo, \& Jhung, 2016). 
Tecnología y

Ciencias $₫$ Agua
2021, Instituto Mexicano de Tecnología del Agua

Open Access bajo la licencia CC BY-NC-SA 4.0

(https://creativecommons.org/licenses/by-nc-sa/4.0/)

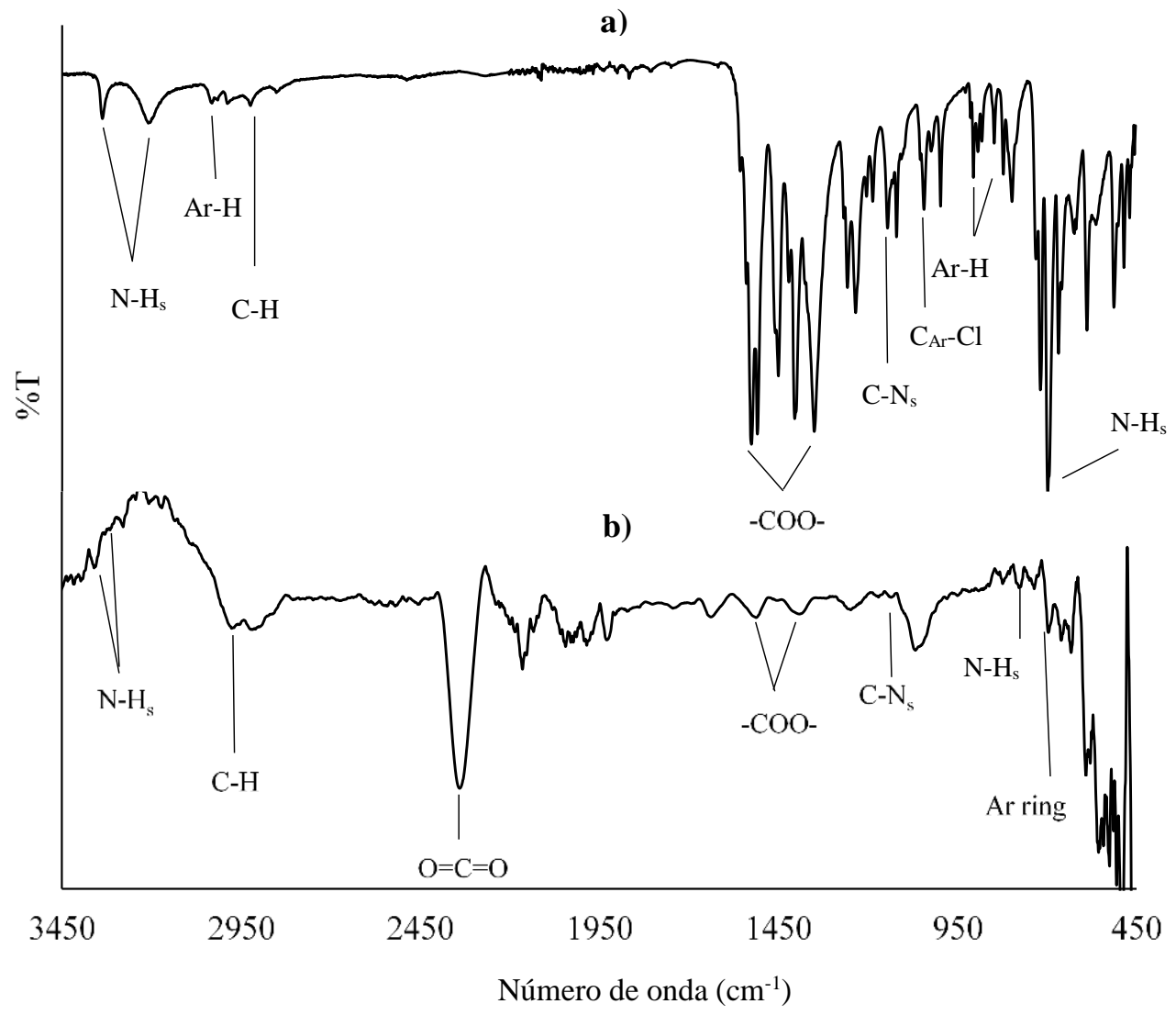

Figura 2. Espectro de infrarrojo del diclofenaco de sodio (Sigma Aldrich, CAS: 15307-79-6 ): a) sólido, b) solución acuosa.

Además, se realizó el análisis del COT, carbono total (CT), $\mathrm{DBO}_{5}$, DQO y se calculó el índice de biodegradabilidad (IBD) como se muestra en la Tabla 1. De acuerdo con Fajardo y Córdoba (2005), si el IBD $\left(\mathrm{DBO}_{5} / \mathrm{DQO}\right)$ es $<0.2$, la materia orgánica presente en el agua es muy poco biodegradable, mientras que si los valores son $>0.6$ es biodegradable. De acuerdo con lo anterior, en la Tabla 1 se observa que 
2021, Instituto Mexicano de Tecnología del Agua

Open Access bajo la licencia CC BY-NC-SA 4.0

(https://creativecommons.org/licenses/by-nc-sa/4.0/)

las tres concentraciones analizadas tienen un IBD $\leq 0.2$; a medida que la concentración de DCF aumenta, el índice de biodegradabilidad disminuye.

Tabla 1. Caracterización inicial de las soluciones acuosas de diclofenaco.

\begin{tabular}{|c|c|c|c|c|c|}
\hline $\begin{array}{c}\text { Concentración } \\
\mathbf{( m g / I )}\end{array}$ & $\begin{array}{c}\text { COT } \\
\mathbf{( m g / I )}\end{array}$ & $\begin{array}{c}\text { CT } \\
\mathbf{( m g / I )}\end{array}$ & $\begin{array}{c}\text { DQO } \\
\mathbf{( m g / I )}\end{array}$ & $\begin{array}{c}\text { DBO }_{5} \\
\mathbf{( m g / I )}\end{array}$ & IBD \\
\hline 10 & 4.87 & 6.02 & 15.25 & 4.34 & 0.21 \\
\hline 50 & 26.55 & 31.94 & 60.43 & 2.59 & 0.04 \\
\hline 150 & 70.70 & 93.50 & 180.36 & 1.96 & 0.01 \\
\hline
\end{tabular}

Fluorescencia

La Figura 3 muestra la matriz de excitación-emisión (EEM) del análisis de la solución de DCF, donde se observan dos picos de fluorescencia mayores (Pico A y B) y tres picos menores. El pico A tuvo una $\Lambda_{\text {exc }} / \Lambda_{\text {em }}$ de $240 / 362.5$ nm y una intensidad de fluorescencia (IF) de 299.64 Unidades Arbitrarias de Fluorescencia (UAF). El pico B tuvo una $\Lambda_{\text {exc }} / \Lambda_{\mathrm{em}}$ de 285/364.5 nm y una IF de $355.54 \mathrm{UAF}$. Los tres picos menores se localizaron a una $\Lambda_{\text {exc }} / \Lambda_{\mathrm{em}}$ de $245 / 420,250 / 440$ y $320 / 365$ nm, respectivamente. 
Tecnología y

Ciencias $₫$ Agua
2021, Instituto Mexicano de Tecnología del Agua

Open Access bajo la licencia CC BY-NC-SA 4.0

(https://creativecommons.org/licenses/by-nc-sa/4.0/)

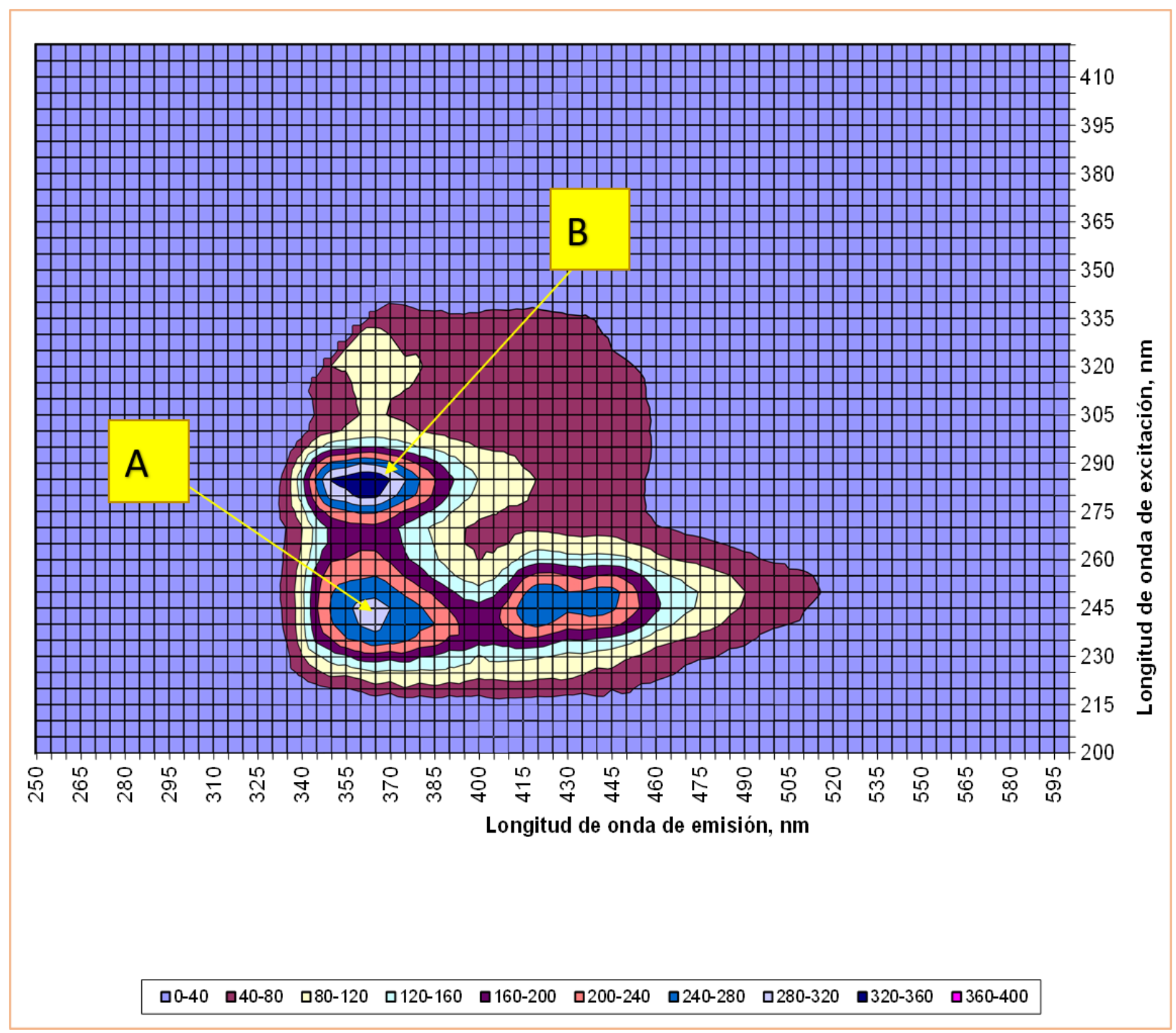

Figura 3. EEM de DCF a $150 \mathrm{mg} / \mathrm{l}$. 


\section{Proceso de electro-oxidación (EO)}

Los resultados del proceso de EO se muestran en la Tabla 2, aplicando diferentes combinaciones de las variables de estudio en los ocho experimentos realizados por triplicado a $150 \mathrm{mg} / \mathrm{I}$ de DCF y $360 \mathrm{~min}$ de tratamiento.

Tabla 2. Resultados del proceso de electro-oxidación de diclofenaco.

\begin{tabular}{|c|c|c|c|c|c|c|c|c|c|c|}
\hline & $\mathbf{A}$ & $\mathbf{B}$ & $\mathbf{C}$ & $\mathbf{Y}$ & $\mathbf{p H o}$ & $\mathbf{p H} \mathbf{T}$ & $\begin{array}{c}\text { Tiempo } \\
\mathbf{m i n}\end{array}$ & $\begin{array}{c}\text { \% de } \\
\text { degradación }\end{array}$ & Fo & Probabilidad \\
\hline $\mathbf{1}$ & 0.5 & $\mathrm{No}$ & $\mathrm{Na}_{2} \mathrm{SO}_{4}$ & 13.2 & 6.36 & 4.18 & 360 & 90.5 & 5.25 & 0 \\
\hline $\mathbf{2}$ & 1.0 & $\mathrm{No}$ & $\mathrm{Na}_{2} \mathrm{SO}_{4}$ & 28.9 & 5.70 & 3.15 & 360 & 81 & 0.59 & 0.452 \\
\hline $\mathbf{3}$ & 0.5 & $\mathrm{Si}$ & $\mathrm{Na}_{2} \mathrm{SO}_{4}$ & 3.0 & 5.90 & 3.83 & 360 & 98 & 0.25 & 0.627 \\
\hline $\mathbf{4}$ & 1.0 & $\mathrm{Si}$ & $\mathrm{Na}_{2} \mathrm{SO}_{4}$ & 19.6 & 6.30 & 2.31 & 360 & 87.2 & 3.88 & 0.067 \\
\hline $\mathbf{5}$ & 0.5 & $\mathrm{No}$ & $\mathrm{NaCl}$ & 32.8 & 5.29 & 6.71 & 360 & 77.7 & 1.99 & 0.178 \\
\hline $\mathbf{6}$ & 1.0 & $\mathrm{No}$ & $\mathrm{NaCl}$ & 3.3 & 6.10 & 6.31 & 360 & 97.8 & 7.89 & 0.013 \\
\hline $\mathbf{7}$ & 0.5 & $\mathrm{Si}$ & $\mathrm{NaCl}$ & 33.6 & 5.94 & 6.41 & 360 & 77.1 & 18.38 & 0.001 \\
\hline $\mathbf{8}$ & 1.0 & $\mathrm{Si}$ & $\mathrm{NaCl}$ & 28.4 & 6.38 & 7.06 & 360 & 80.3 & 3.77 & 0.07 \\
\hline
\end{tabular}

$\mathrm{A}=$ intensidad de corriente; $\mathrm{B}=$ luz UV; $\mathrm{C}=$ electrolito soporte; $\mathrm{Y}=$ concentración de DCF medida por UV-Vis a $276 \mathrm{~nm}$.

De acuerdo con los resultados obtenidos en la Tabla 2, se observa que las mejores condiciones se tuvieron al aplicar una intensidad de 
corriente de $0.5 \mathrm{~A}\left(\mathrm{~J}=12.64 \mathrm{~A} / \mathrm{m}^{2}\right)$ en presencia de luz UV y usando $\mathrm{Na}_{2} \mathrm{SO}_{4}$ como electrolito soporte, obteniendo un $98 \%$ de mineralización. Por otro lado, en el experimento 6 , donde el proceso se llevó a cabo a 1.0 A $\left(\mathrm{J}=25.29 \mathrm{~A} / \mathrm{m}^{2}\right), \mathrm{NaCl}$ y ausencia de luz UV, se obtuvo un porcentaje de mineralización similar de $97.8 \%$. Para el análisis de los resultados se evaluó el efecto de cada una de las variables implicadas en el proceso.

\section{Efecto del electrolito soporte}

Los experimentos se efectuaron con pH inicial de 5.29-6.38. Se observó que al utilizar $\mathrm{Na}_{2} \mathrm{SO}_{4}$ como electrolito soporte, el pH disminuyó hasta 2.31 (Tabla 2). De acuerdo con Brillas, Garcia-Segura, Skoumal y Arias (2010), a un $\mathrm{pH}=2-3$, es posible que se produzca un proceso de precipitaciónredisolución-degradación del fármaco debido a que el pKa del DCF es 4.15 , por lo que al llevar a cabo los experimentos a un pH mayor al pKa se favorece la ionización del mismo; a un pH menor al pKa, el DCF se encuentra en su forma molecular (Brillas et al., 2010). La forma ionizada del DCF es soluble y es la especie electroactiva susceptible a ser oxidada (Brillas et al., 2010; Pérez-Estrada et al., 2005).

La disminución del $\mathrm{pH}$ al utilizar $\mathrm{Na}_{2} \mathrm{SO}_{4}$ como electrolito soporte se explica por la formación del ion persulfato $\left(\mathrm{S}_{2} \mathrm{O}_{8}{ }^{2-}\right)$ (Ecuación (1)), el cual puede hidrolizarse para formar el ion peroxomonosulfato $\left(\mathrm{HSO}_{5}{ }^{-}\right)$y el ion 
bisulfato $\left(\mathrm{HSO}_{4}^{-}\right)$, como se muestra en la Ecuación (2) (Ross \& Neta, 1982).

$2 \mathrm{SO}_{4}^{2-} \rightarrow \mathrm{S}_{2} \mathrm{O}_{8}^{2-}+2 e^{-}$

$\mathrm{S}_{2} \mathrm{O}_{8}^{2-}+\mathrm{H}_{2} \mathrm{O} \rightarrow \mathrm{HSO}_{5}^{-}+\mathrm{HSO}_{4}^{-}$

Los iones $\mathrm{HSO}_{5}^{-}$y $\mathrm{HSO}_{4}^{-}$son anfolitos que en equilibrio entre sí mismos forman sus ácidos conjugados $\left(\mathrm{H}_{2} \mathrm{SO}_{5}\right.$ y $\left.\mathrm{H}_{2} \mathrm{SO}_{4}\right)$ a través de la reacción de dismutación (ecuaciones (3) y (4)):

$$
\begin{aligned}
& \mathrm{HSO}_{5}^{-}+\mathrm{HSO}_{5}^{-} \leftrightarrow \mathrm{H}_{2} \mathrm{SO}_{5}+\mathrm{SO}_{5}^{2-} \\
& \mathrm{HSO}_{4}^{-}+\mathrm{HSO}_{4}^{-} \leftrightarrow \mathrm{H}_{2} \mathrm{SO}_{4}+\mathrm{SO}_{4}^{2-}
\end{aligned}
$$

El ácido peroximonosulfúrico $\left(\mathrm{H}_{2} \mathrm{SO}_{5}\right)$ en pequeñas concentraciones se descompone generando ácido sulfúrico y oxígeno, lo que ocasiona la disminución del $\mathrm{pH}$ a valores de 2-3 por ser un ácido fuerte, $\mathrm{pKa}=-3.0$ (Ecuación (5)):

$$
\mathrm{H}_{2} \mathrm{SO}_{5} \leftrightarrow \mathrm{H}_{2} \mathrm{SO}_{4}+\mathrm{O}_{2}
$$


Tecnología y

Ciencias $₫$ Agua
2021, Instituto Mexicano de Tecnología del Agua

Open Access bajo la licencia CC BY-NC-SA 4.0

(https://creativecommons.org/licenses/by-nc-sa/4.0/)

Esta disminución de $\mathrm{pH}$ puede provocar interferencia en la cuantificación, mostrando la aparente degradación del mismo; sin embargo, sólo se encuentra precipitado, razón por la cual es necesaria una buena elección del electrolito soporte o bien tener un control del pH mediante un buffer. Al utilizar $\mathrm{Na}_{2} \mathrm{SO}_{4}$, el mejor porcentaje de remoción fue de $98 \%$ a $0.5 \mathrm{~A}\left(\mathrm{~J}=12.64 \mathrm{~A} / \mathrm{m}^{2}\right)$ y en presencia de luz UV (Figura $4)$; este resultado podría atribuirse a la formación de oxidantes como el persulfato $\left(\mathrm{S}_{2} \mathrm{O}_{8}^{2-}\right)$ sin embargo, las especies que forma este radical al estar en solución acuosa propicia un medio ácido $\left(\mathrm{H}_{2} \mathrm{SO}_{4}\right)$, lo cual ocasiona el fenómeno de precipitación-redisolución-degradación del DCF antes mencionado.

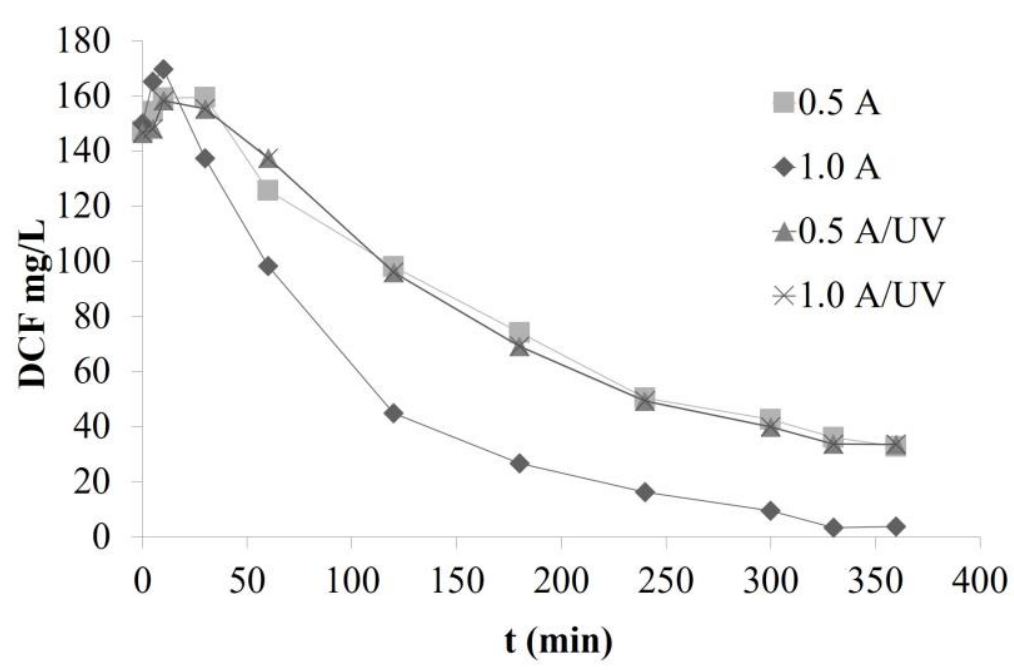

a)

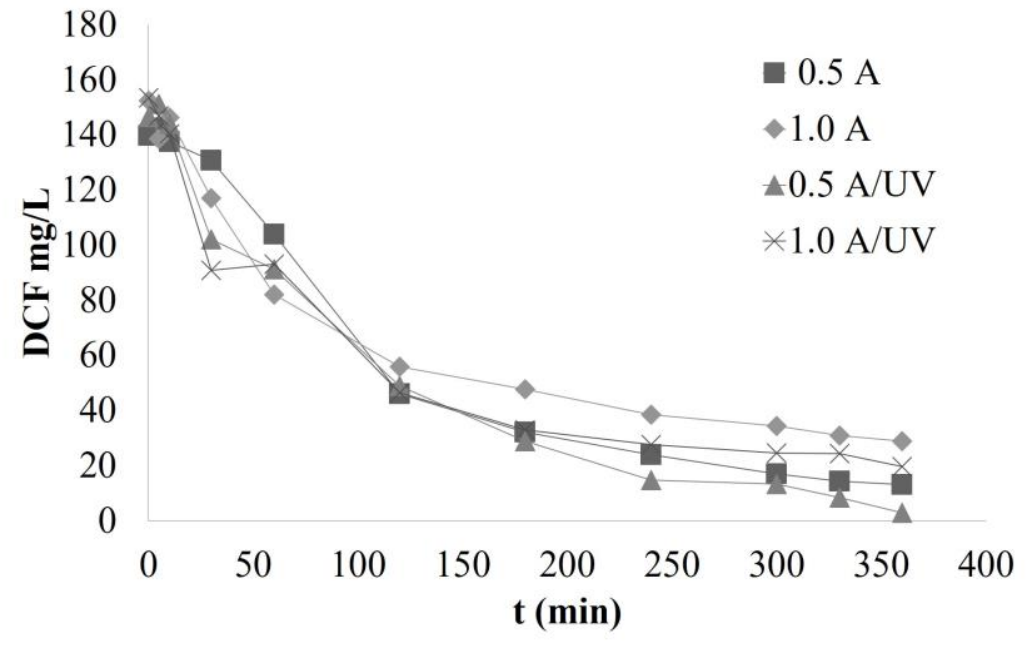

b)

Figura 4. Efecto del electrolito soporte: a) $\mathrm{NaCl}$ y b) $\mathrm{Na}_{2} \mathrm{SO}_{4}$. 
Por otra parte, al utilizar $\mathrm{NaCl}$ se mantiene el valor de $\mathrm{pH}$ y se genera el ion $\mathrm{Cl}^{-}$, oxidándose a $\mathrm{Cl}_{2(\mathrm{~g})}$ (Ecuación (6)), favoreciendo la oxidación indirecta (Brillas \& Sirés, 2015). Los experimentos subsecuentes se realizaron de acuerdo con las condiciones de operación establecidas en el experimento 6, para evitar interferencias en la cuantificación del mismo:

$2 \mathrm{Cl}^{-} \rightarrow \mathrm{Cl}_{2(g)}+2 \mathrm{e}^{-}$

Cabe mencionar que de acuerdo con las investigaciones realizadas por Brillas y Sirés (2015), la oxidación mediada con cloro activo se vuelve más rápida en medios ácidos que en alcalinos debido al potencial estándar del $\mathrm{Cl}_{2}\left(\mathrm{E}^{\circ}=1.36 \mathrm{~V} / \mathrm{SHE}\right)$. Sin embargo, el mayor inconveniente de este procedimiento es que es susceptible de generar cloroderivados tóxicos, trihalometanos y cloraminas, que pueden aumentar la toxicidad del efluente. Al utilizar $\mathrm{NaCl}$ como electrolito soporte y una intensidad de corriente de $1.0 \mathrm{~A}\left(\mathrm{~J}=25.29 \mathrm{~A} / \mathrm{m}^{2}\right)$ se obtiene una eficiencia de remoción de $97.8 \%$.

\section{Efecto de la intensidad de corriente}


2021, Instituto Mexicano de Tecnología del Agua

Open Access bajo la licencia CC BY-NC-SA 4.0

(https://creativecommons.org/licenses/by-nc-sa/4.0/)

La intensidad de corriente aplicada es uno de los parámetros con mayor importancia en la electroquímica, ya que controla la transferencia de electrones y la generación de reactivos oxidantes lo cual influye directamente en las eficiencias de remoción de los contaminantes (Gurung, Chaker-Ncibi, Shestakova, \& Sillanpää, 2018).En la Figura 5 se observa que al aplicar una intensidad de corriente de 1 A $(\mathrm{J}=25.29$ $\left.A / \mathrm{m}^{2}\right), \mathrm{NaCl}$ y en ausencia de luz UV se obtiene el $97.8 \%$ de remoción de DCF; mientras que a $0.5 \mathrm{~A}\left(\mathrm{~J}=12.64 \mathrm{~A} / \mathrm{m}^{2}\right)$ se obtiene un porcentaje de degradación de $77.7 \%$, mostrando que a mayor intensidad de corriente, mayor porcentaje de remoción. Esto se debe a que al aumentar la corriente aplicada se presenta una mayor generación de $\mathrm{H}_{2} \mathrm{O}_{2}$, lo que induce una mayor producción de radicales hidroxilo (Jaafarzadeh, Ghanbari, \& Moradi, 2015). Por otro lado, cuando se aplicaron 0.5 A se observó una notable diferencia entre el uso de cada uno de los electrolitos soporte; ya que con $\mathrm{Na}_{2} \mathrm{SO}_{4}$ se obtuvieron mejores resultados (90.5 y $98 \%$ en ausencia y presencia de luz UV, respectivamente), esto se relaciona con la producción de radicales persulfato que ayudan a la oxidación del DCF a intensidades de corriente más bajas que con $\mathrm{NaCl}$; no obstante, al aumentar la intensidad a $1 \mathrm{~A}\left(\mathrm{~J}=25.29 \mathrm{~A} / \mathrm{m}^{2}\right)$, la eficiencia del proceso disminuye, alcanzando solamente un $81 \%$ para los experimentos en ausencia de luz UV y $87.2 \%$ en presencia de radiación UV. Dicho fenómeno se debe a que al aumentar la intensidad de corriente se produce la descomposición del peróxido de hidrógeno en el ánodo, lo cual ocasiona la aparición de reacciones parásitas entre los radicales $\bullet^{\circ} \mathrm{OH}$, generando $\mathrm{O}_{2}$ gas o la dimerización de dichos radicales, lo cual afecta 
Tecnología y

Ciencias $₫$ Agua
2021, Instituto Mexicano de Tecnología del Agua

Open Access bajo la licencia CC BY-NC-SA 4.0

(https://creativecommons.org/licenses/by-nc-sa/4.0/)

negativamente a las propiedades oxidativas del radical $\bullet \mathrm{OH}$ (Jaafarzadeh et al., 2015; Yang et al., 2018).

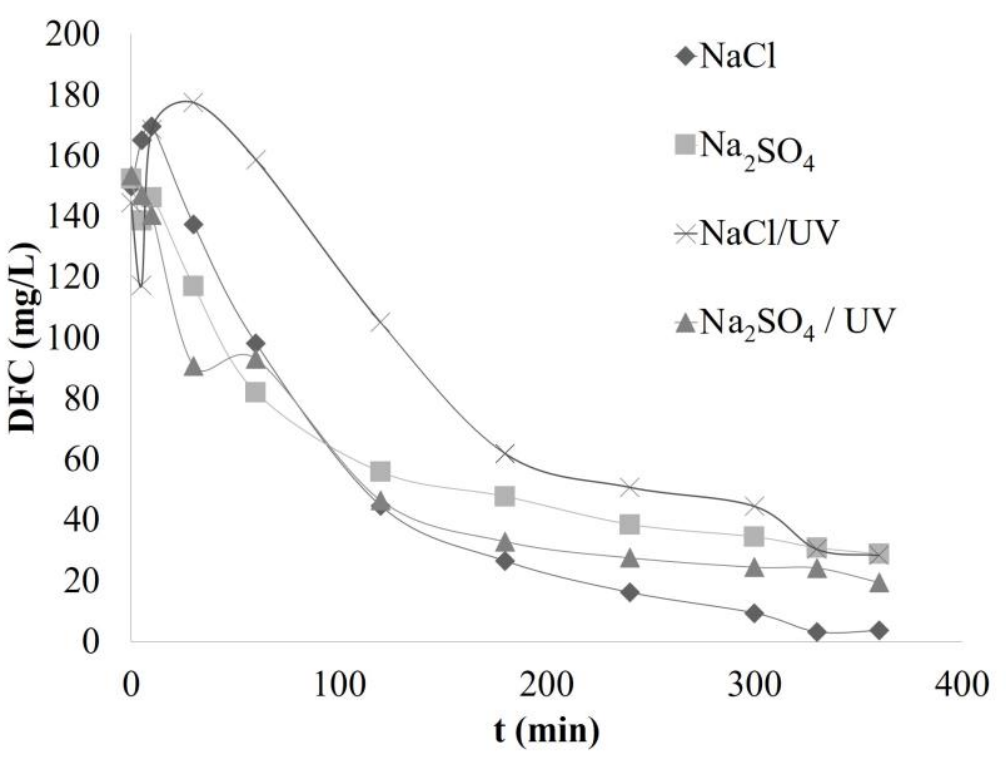

a)

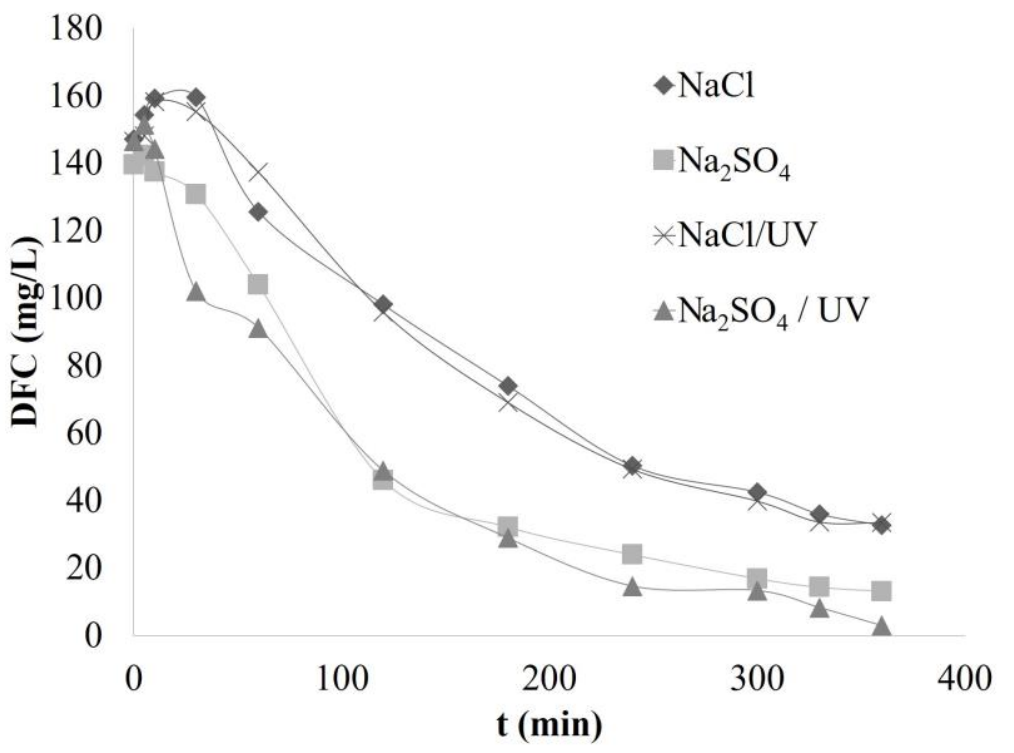

b)

Figura 5. Efecto de la intensidad de corriente: a) $1.0 \mathrm{~A} \mathrm{y} \mathrm{b)} 0.5 \mathrm{~A}$.

De acuerdo con el análisis de ANOVA se puede observar que el valor de $F_{0}(0.25)$ para la variable de intensidad de corriente se encuentra dentro del valor de aceptación (5.25), lo cual indica que genera un efecto importante en el proceso de electro-oxidación.

Además se calculó el consumo energético del sistema durante la electro-oxidación (Tabla 3). De acuerdo con la Comisión Federal de Electricidad (CFE) para la ciudad de Toluca, el costo de cada kWh es de $\$ 0.58$, por lo tanto los experimentos realizados a $1.0 \mathrm{~A}\left(\mathrm{~J}=25.29 \mathrm{~A} / \mathrm{m}^{2}\right)$ 
tienen un costo de $\$ 31.8$ por $\mathrm{m}^{3}$, mientras que a $0.5 \mathrm{~A}\left(\mathrm{~J}=12.64 \mathrm{~A} / \mathrm{m}^{2}\right)$ de $\$ 12.0$ por $\mathrm{m}^{3}$; estos valores se calcularon a $180 \mathrm{~min}$.

Tabla 3. Costo energético.

\begin{tabular}{|c|c|c|c|c|}
\hline $\begin{array}{c}\text { Intensidad } \\
\text { de corriente } \\
\text { (A) }\end{array}$ & $\begin{array}{c}\text { Voltaje } \\
\text { (V) }\end{array}$ & $\begin{array}{c}\text { Tiempo de } \\
\text { experimento } \\
(\mathrm{min})\end{array}$ & $\begin{array}{c}\text { Consumo } \\
\text { energético } \\
\left(\mathrm{kW} / \mathrm{m}^{3}\right)\end{array}$ & $\begin{array}{c}\text { Costo } \\
\text { energético } \\
\left(\mathrm{MNX} / \mathrm{m}^{3}\right)\end{array}$ \\
\hline 0.5 & 5.5 & 180 & 20.63 & 12.0 \\
\hline 1.0 & 7.3 & 180 & 54.75 & 31.8 \\
\hline
\end{tabular}

\section{Efecto de la luz UV}

La irradiación UV tiene como principal función romper los enlaces químicos cuando la energía fotónica absorbida excede la energía de enlace. Cuando se lleva a cabo la ruptura del enlace, un electrón desapareado permanece en cada fragmento teniendo como resultado la formación de radicales. En los compuestos clorados, como el diclofenaco, bajo radiación UV pueden romper un enlace de $\mathrm{C}-\mathrm{Cl}$ relativamente débil (energía de disociación de enlace $=330 \mathrm{~kJ} / \mathrm{mol}$ ), que da como resultado la formación de un radical de cloro $(\mathrm{Cl} \bullet)$ y un electrón desapareado en el carbono de la molécula orgánica, MO-C• (Keen, Thurman, Ferrer, Dotson, \& Lindena, 2013). 
Tecnología y

Ciencias $₫$ Agua
2021, Instituto Mexicano de Tecnología del Agua

Open Access bajo la licencia CC BY-NC-SA 4.0

(https://creativecommons.org/licenses/by-nc-sa/4.0/)

El porcentaje de degradación al usar $\mathrm{NaCl}$ en los experimentos realizados a $0.5 \mathrm{~A}\left(\mathrm{~J}=12.64 \mathrm{~A} / \mathrm{m}^{2}\right)$ en ausencia y presencia de luz UV son similares (77.7 y $77.1 \%$, respectivamente). Por otra parte, en los experimentos a $1 \mathrm{~A}\left(\mathrm{~J}=25.29 \mathrm{~A} / \mathrm{m}^{2}\right)$ en presencia de luz $\mathrm{UV}$, se obtuvo el $80.3 \%$ de mineralización y en ausencia de luz el $97.8 \%$ (Figura 6). Este fenómeno se atribuye a que en procesos de EO con electrolitos como el $\mathrm{NaCl}$ y electrodos de DDB se producen altos niveles de cloro activo electro generado, especie que posee propiedades oxidativas, sin embargo este fenómeno disminuye cuando se presenta una incidencia de luz UV (Hurwitz, Pornwongthong, Mahendra, \& Hoek,2014; Sánchez-Montes et al., 2020).

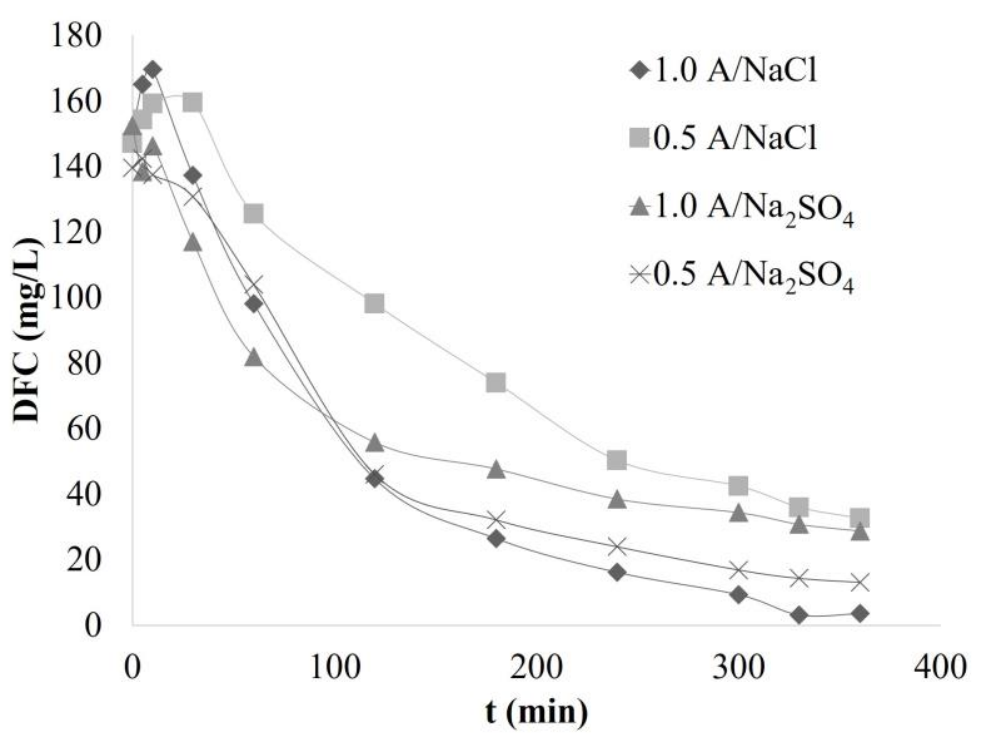

a)

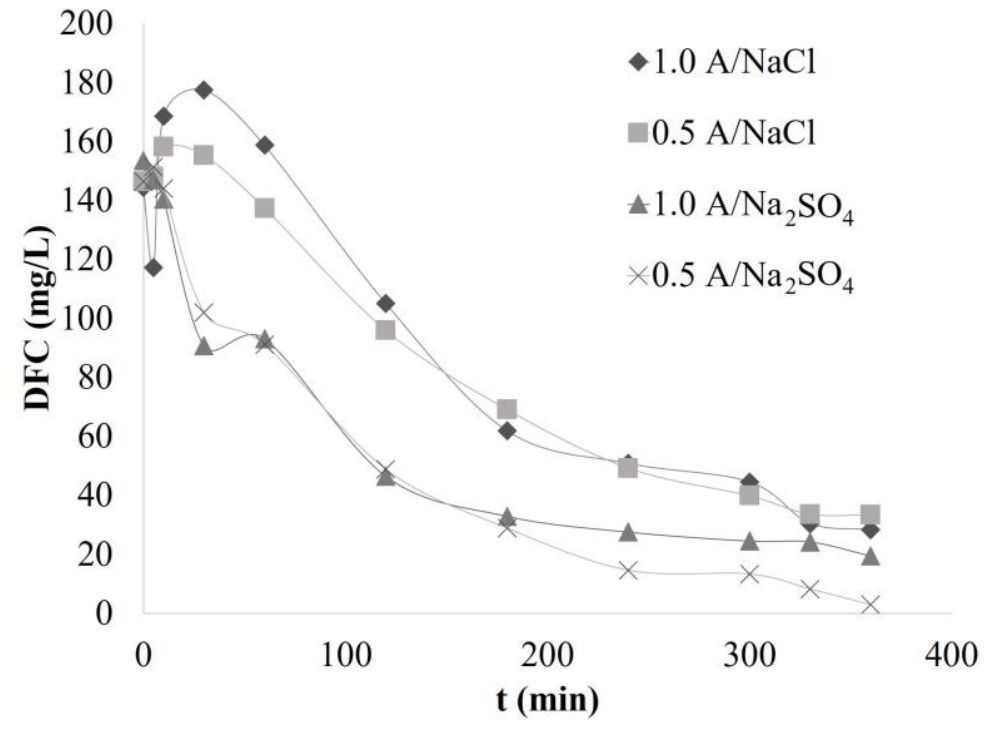

b)

Figura 6. Efecto de la luz UV en la electro-oxidación de diclofenaco: a) ausencia de luz y b) presencia de luz UV. 
De acuerdo con Sánchez-Montes et al. (2020), el proceso de EO en presencia de luz UV -electrodos con una amplia ventana electroquímica anódica, como el DDB y electrolitos soporte clorados- permite la formación de especies de $\mathrm{ClO}_{3}^{-}$y $\mathrm{ClO}_{4}^{-}$, en donde la concentración de $\mathrm{ClO}_{3}{ }^{-}$ es ligeramente mayor en presencia de luz UV que en su ausencia; si bien el $\mathrm{ClO}_{3}{ }^{-}$es una especie oxidante, su bajo potencial redox ( $\mathrm{E}^{\circ}=0.35 \mathrm{~V}$ ) en soluciones con un $\mathrm{pH}$ de entre 6 y 7 provoca una disminución en la eficiencia del proceso (Hurwitz et al., 2014; Sánchez-Montes et al., 2020).

Por otro lado, con $\mathrm{Na}_{2} \mathrm{SO}_{4}$ se presenta un mayor porcentaje de degradación en presencia de luz UV debido a que la radiación propicia la generación del radical sulfato a partir del ion persulfato formado en el proceso de oxidación (Ecuación (7)), el cual tiene un alto potencial redox $\left(E^{\circ}=2.5-3.1 \mathrm{~V}\right)$. Dichos radicales sulfato tienden a ser más selectivos que - OH para la oxidación de contaminantes orgánicos (Lu et al., 2017):

$\mathrm{S}_{2} \mathrm{O}_{8}^{2-}+h v \rightarrow 2 \mathrm{SO}_{4}^{\bullet}$

Con base en los resultados obtenidos en el ANOVA, se observa que los experimentos realizados tuvieron un valor $p=0.001$, lo cual nos indica que existe una diferencia estadísticamente significativa entre éstos.

\section{Efecto de la concentración inicial}


En la Figura 7 se muestran los barridos de UV-Vis que se obtuvieron durante los experimentos realizados a diferentes concentraciones (150, 50 y $10 \mathrm{mg} / \mathrm{l}$ ). Se observa una disminución gradual en el experimento a $10 \mathrm{mg} / \mathrm{l}$, teniendo así una eficiencia del $100 \%$ a $330 \mathrm{~min}$; mientras que a $150 \mathrm{mg} / \mathrm{l}$ se observa que la primera hora de tratamiento es la más eficiente obteniendo más del 50 \% de mineralización. De acuerdo con lo anterior, al incrementar la concentración del fármaco, el tiempo de tratamiento es mayor y la eficiencia disminuye. 
Tecnología y

Ciencias $₫$ Agua

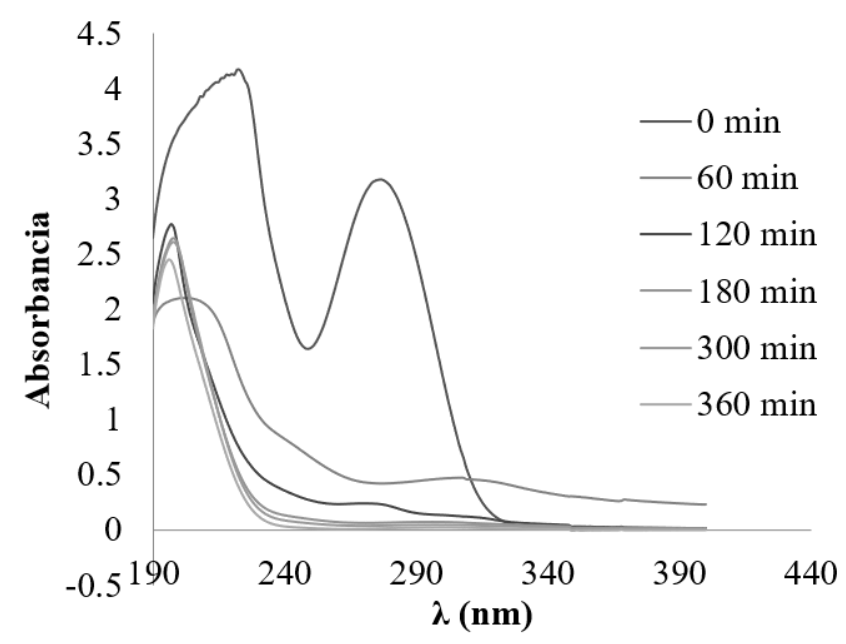

a)
2021, Instituto Mexicano de Tecnología del Agua

Open Access bajo la licencia CC BY-NC-SA 4.0

(https://creativecommons.org/licenses/by-nc-sa/4.0/)

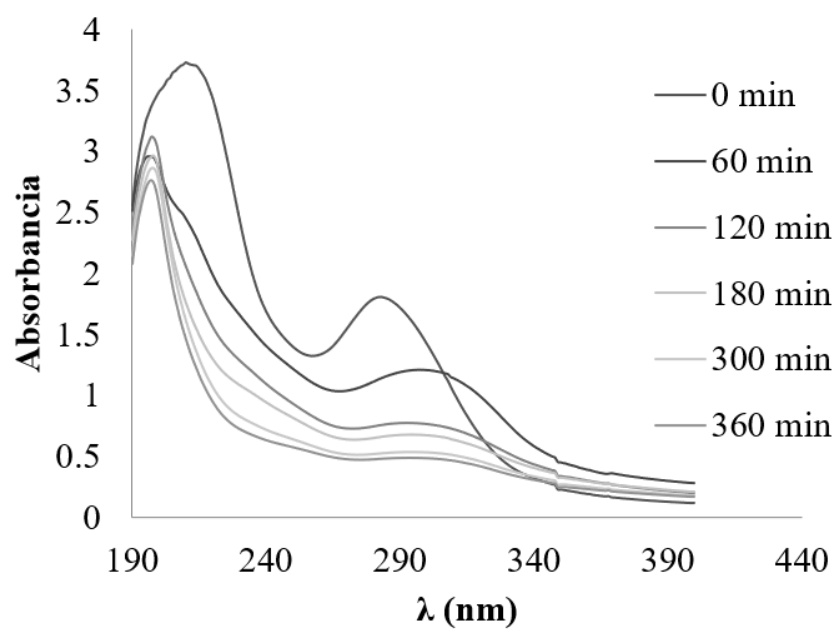

b)

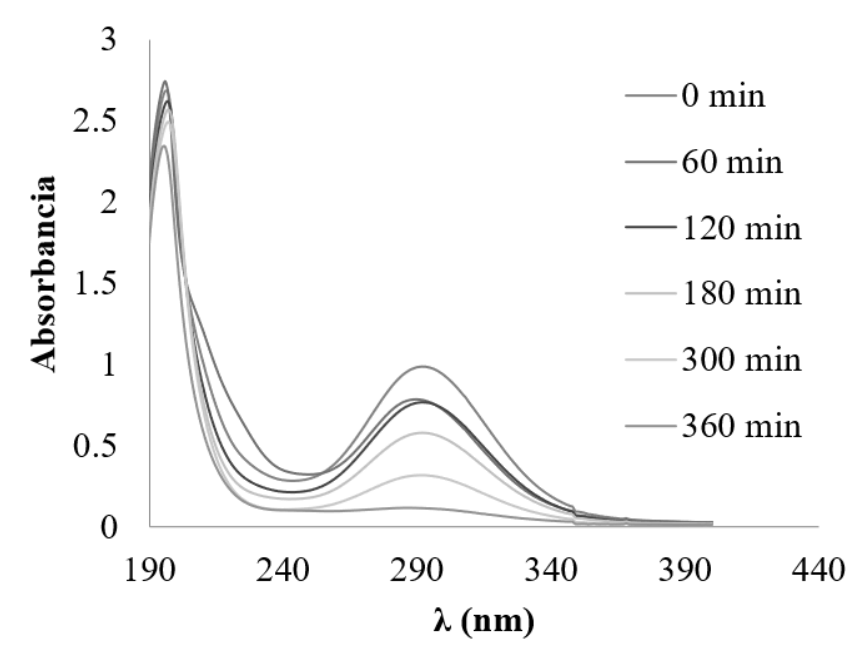

c)

Figura 7. Barrido UV-Vis de diclofenaco a $1.0 \mathrm{~A}$ : a) $150 \mathrm{mg} / \mathrm{l}$, b) 50 $\mathrm{mg} / \mathrm{l}$ y c) $10 \mathrm{mg} / \mathrm{l}$. 
Tecnología y

Ciencias $₫$ Agua
2021, Instituto Mexicano de Tecnología del Agua

Open Access bajo la licencia CC BY-NC-SA 4.0

(https://creativecommons.org/licenses/by-nc-sa/4.0/)

Con base en los resultados, las mejores condiciones de oxidación de diclofenaco fueron $\mathrm{NaCl}$ como electrolito soporte, $1.0 \mathrm{~A}\left(\mathrm{~J}=25.29 \mathrm{~A} / \mathrm{m}^{2}\right)$ y ausencia de luz UV, las cuales se utilizaron en concentraciones de 10 y 50 mg/l de DCF. En la Tabla 4 se muestran los valores de COT, DQO, $\mathrm{DBO}_{5}$ y HPLC obtenidos antes y después del tratamiento, en donde se puede observar que en términos de $\mathrm{DQO}^{\text {y }} \mathrm{DBO}_{5}$ se presenta una eficiencia del $100 \%$, con excepción de la concentración de 150 mg/l en donde en términos de DQO se obtiene una eficiencia del $89.3 \%$, indicando que aún hay subproductos susceptibles de ser oxidados por medios químicos.

Tabla 4. Caracterización inicial y final del diclofenaco.

\begin{tabular}{|c|c|c|c|c|c|c|c|c|c|}
\hline \multicolumn{2}{|c|}{$\begin{array}{c}\text { Muestra } \\
(\mathrm{mg} / \mathrm{l})\end{array}$} & $\begin{array}{l}\text { UV-Vis } \\
(\mathrm{mg} / \mathrm{I})\end{array}$ & $\begin{array}{c}\text { COT } \\
(\mathrm{mg} / \mathrm{l})\end{array}$ & $\begin{array}{l}\mathrm{DBO}_{5} \\
(\mathrm{mg} / \mathrm{l})\end{array}$ & $\begin{array}{c}\text { DQO } \\
(\mathrm{mg} / \mathrm{l})\end{array}$ & $\begin{array}{l}\text { HPLC } \\
(\mathrm{mg} / \mathrm{l})\end{array}$ & $\begin{array}{l}\% \text { DFC } \\
\text { UV-Vis }\end{array}$ & $\begin{array}{l}\% \text { DFC } \\
\text { HPLC }\end{array}$ & $\%$ COT \\
\hline \multirow{2}{*}{10} & Inicial & 10.7 & 4.34 & 4.87 & 15.25 & 10.7 & \multirow{2}{*}{99.98} & \multirow{2}{*}{99.64} & \multirow{2}{*}{63.7} \\
\hline & Final & 0.108 & 0.00 & 1.77 & 0.00 & 0.48 & & & \\
\hline \multirow{2}{*}{50} & Inicial & 50.3 & 26.55 & 2.59 & 60.43 & 50.3 & \multirow{2}{*}{99.08} & \multirow{2}{*}{98.76} & \multirow{2}{*}{43.4} \\
\hline & Final & 0.46 & 0.00 & 15.03 & 0.00 & 0.62 & & & \\
\hline \multirow{2}{*}{150} & Inicial & 150.7 & 70.70 & 1.96 & 180.36 & 150.7 & \multirow{2}{*}{99.99} & \multirow{2}{*}{95.15} & \multirow{2}{*}{64.4} \\
\hline & Final & 0.010 & 0.00 & 12.15 & 13.13 & 0.54 & & & \\
\hline
\end{tabular}

Por otro lado, en términos de COT se obtuvieron las siguientes eficiencias: a una concentración inicial de $150 \mathrm{mg} / \mathrm{l}$, la eficiencia alcanzada fue de $64.4 \%$, mientras que para una concentración inicial de 
50 mg/l se observó el 43.4 \% y para la concentración más baja de 10 $\mathrm{mg} / \mathrm{l}$ se obtuvo el $63.7 \%$.

Los resultados obtenidos por HPLC (Figura 8) mostraron una eficiencia de remoción de 99.64, 98.76 y $95.15 \%$ para las concentraciones de 10,50 y $150 \mathrm{mg} / \mathrm{l}$, respetivamente, a 330 minutos de tratamiento. Estos resultados indican que a mayor concentración de DCF, menor es el porcentaje de remoción. Mientras que en UV-Vis los porcentajes de remoción fueron $99.98,99.08$ y $99.99 \%$ para las mismas concentraciones, respectivamente.

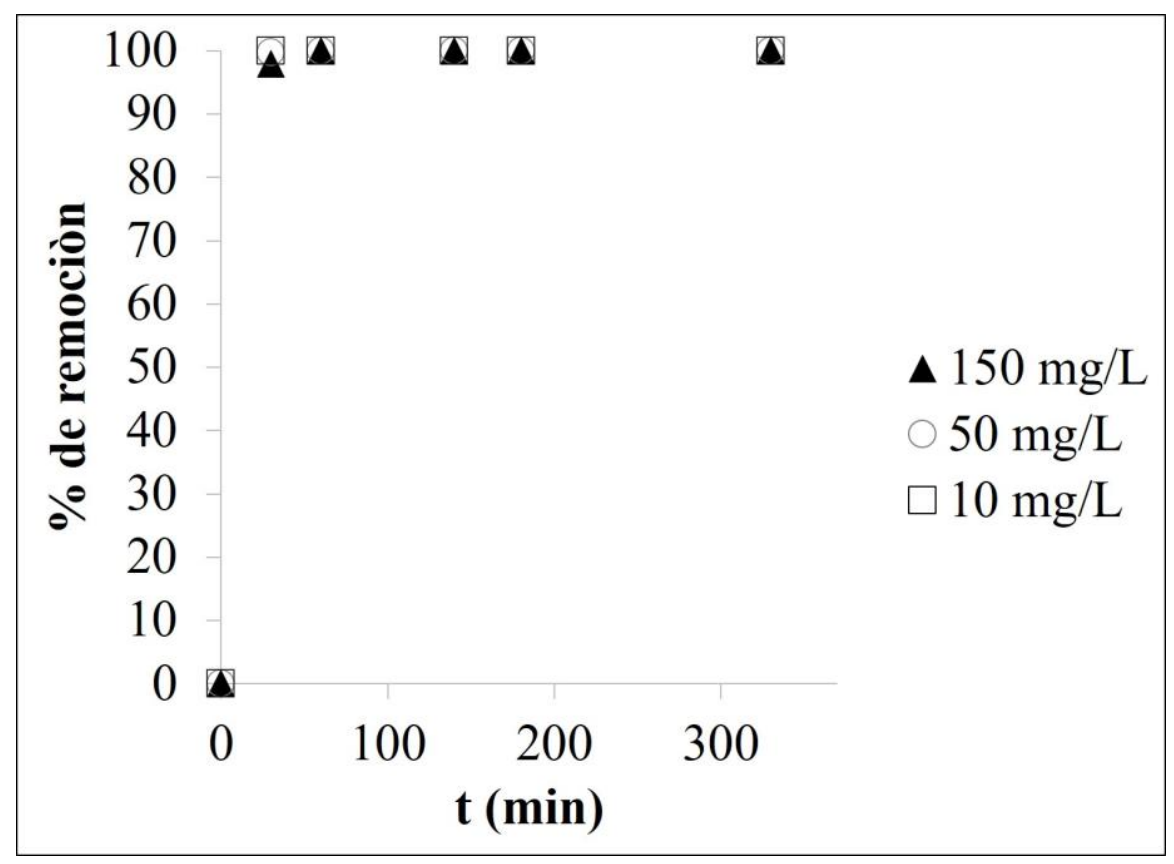

Figura 8. Cinética de electro-oxidación de diclofenaco a diferentes concentraciones (técnica de análisis: HPLC). 
Después del tratamiento el índice de biodegradabilidad aumentó de 0.01 a 0.92 para la concentración de $150 \mathrm{mg} / \mathrm{l}$, lo cual indica que el tratamiento mejora la calidad del efluente.

Después del tratamiento de electro-oxidación se realizaron análisis de IR a las soluciones de DCF de 10, 50 y $150 \mathrm{mg} / \mathrm{l}$ al final del tratamiento (Figura 9). Las bandas después de los $3000 \mathrm{~cm}^{-1}$ desaparecen; las señales correspondientes a $\mathrm{C}=\mathrm{O}$ ácido carboxílico $\left(1242.07 \mathrm{~cm}^{-1}\right), \mathrm{C}-\mathrm{H}$ alifático $\left(2968 \mathrm{~cm}^{-1}\right), \mathrm{C}-\mathrm{Cl}\left(631.69 \mathrm{~cm}^{-1}\right)$ y $\mathrm{Ar}-\mathrm{Cl}\left(1064.72 \mathrm{~cm}^{-1}\right)$ no se observan, lo cual indica la degradación del diclofenaco. También puede observarse la presencia de metabolitos más simples que presentan señales intensas atribuidas a: $\mathrm{CH}_{2}\left(3079.40 \mathrm{~cm}^{-1}\right.$ alargamiento y 674.13 $\mathrm{cm}^{-1}$ deformación); C-Cl alargamiento (806.25, 811.08 y $\left.794.68 \mathrm{~cm}^{-1}\right), y$ la presencia del isómero trans (1640.48 y $1652.05 \mathrm{~cm}^{-1}$ ), lo cual indica la presencia de un compuesto alifático halogenado. 


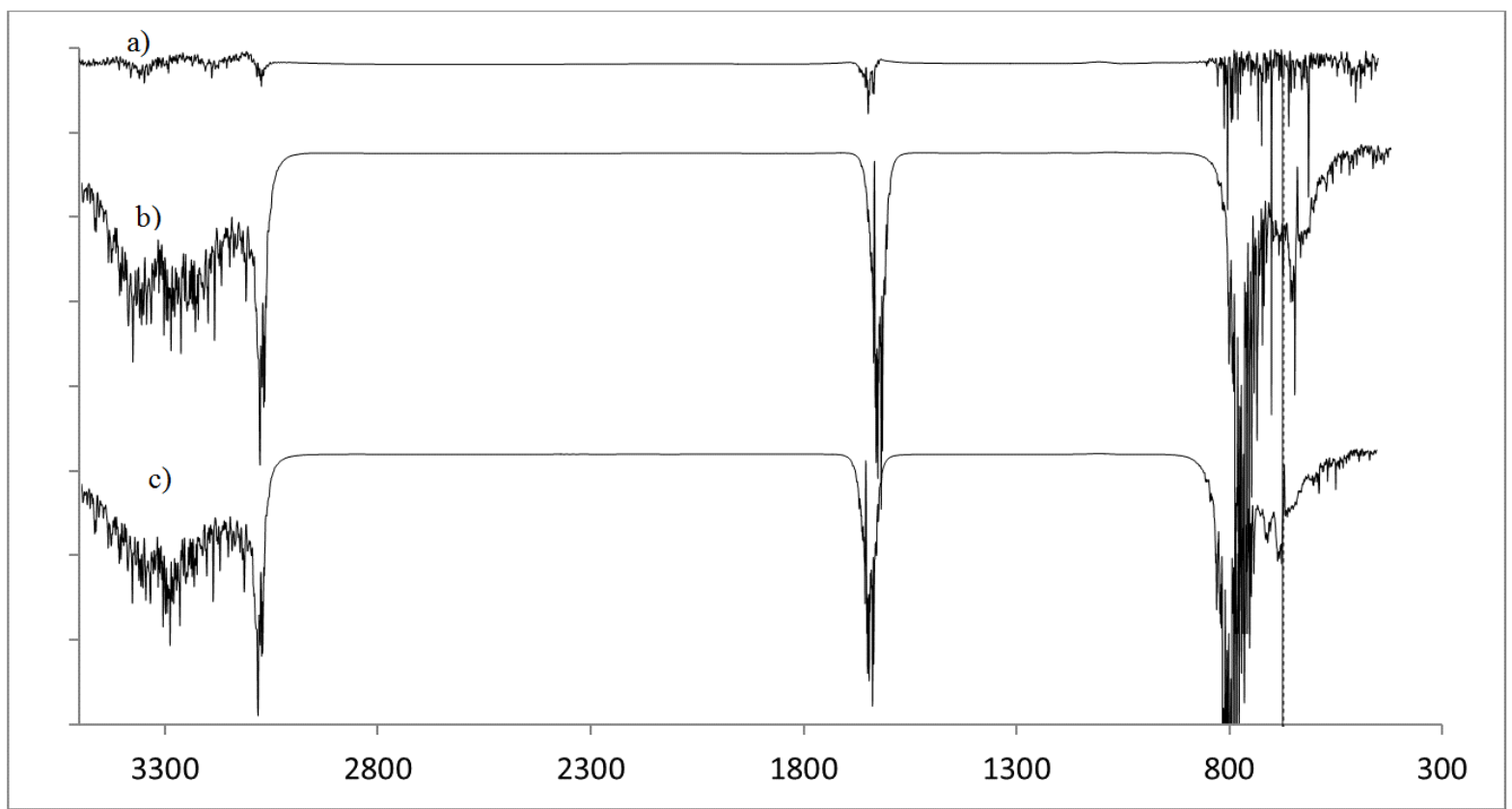

Figura 9. Espectro infrarrojo de las soluciones de diclofenaco después del tratamiento de electro-oxidación: a) $10 \mathrm{mg} / \mathrm{l}$, b) $50 \mathrm{mg} / \mathrm{l}$, c) 150 $\mathrm{mg} / \mathrm{l}$.

El análisis de fluorescencia realizado después del tratamiento de electro-oxidación muestra una disminución en los valores de las longitudes de onda. La $\Lambda_{\mathrm{em}}$ de los picos A y B se desplazó a longitudes de onda más larga después del tratamiento (de 362.5-364.5 nm a 396.5$381.5 \mathrm{~nm}$ ). Por otro lado, la kex se desplazó a longitudes de onda más corta después del tratamiento. El movimiento de los picos A y B podría haber sido causado por la degradación del diclofenaco durante la electrooxidación. Sin embargo, cuando la concentración inicial fue de 150 mg/l se observó un incremento en la IF de la muestra; esto se debe a que la muestra final no se oxida por completo (Figura 10). 
Ciencias $₫$ Agua

2021, Instituto Mexicano de Tecnología del Agua

Open Access bajo la licencia CC BY-NC-SA 4.0

(https://creativecommons.org/licenses/by-nc-sa/4.0/)

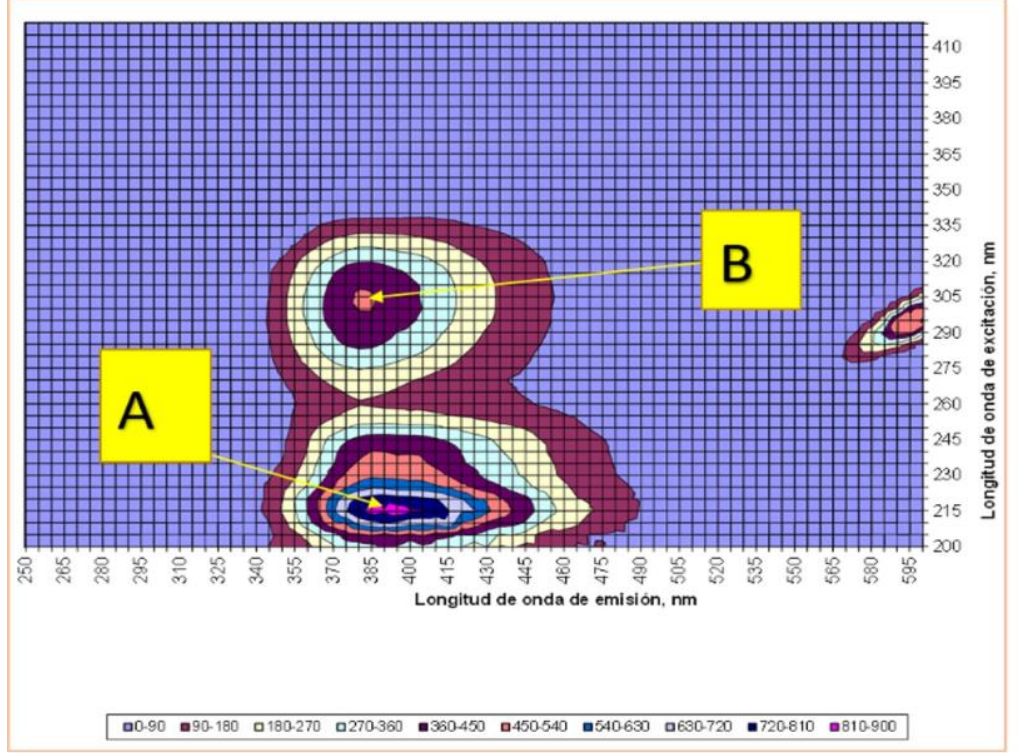

a)

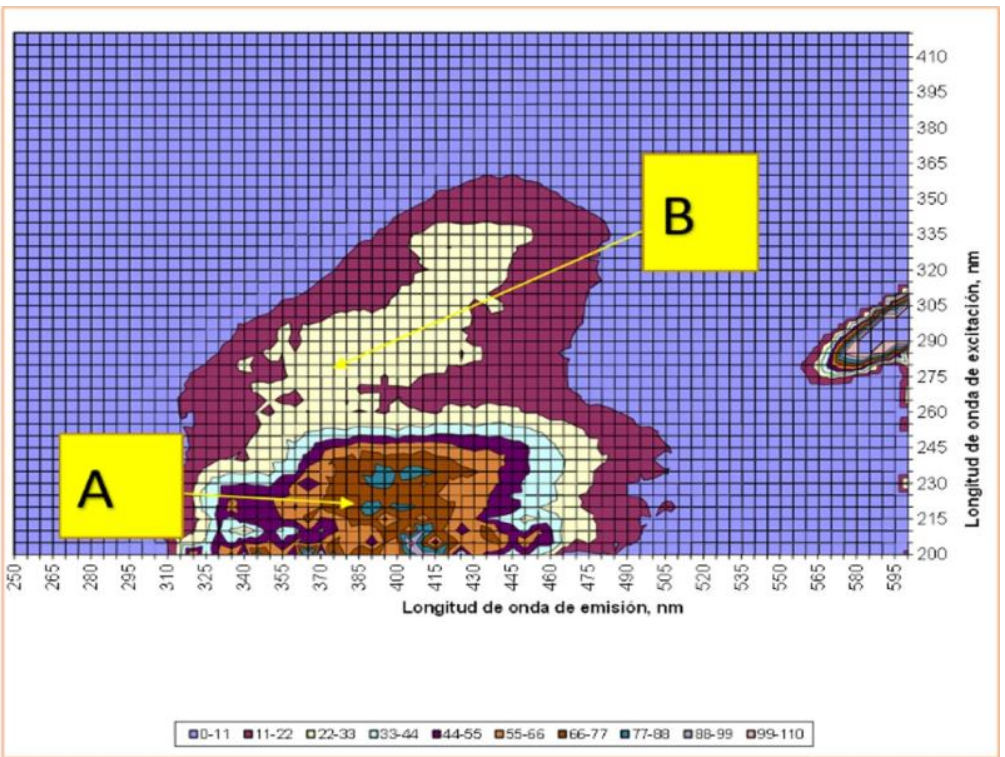

b)

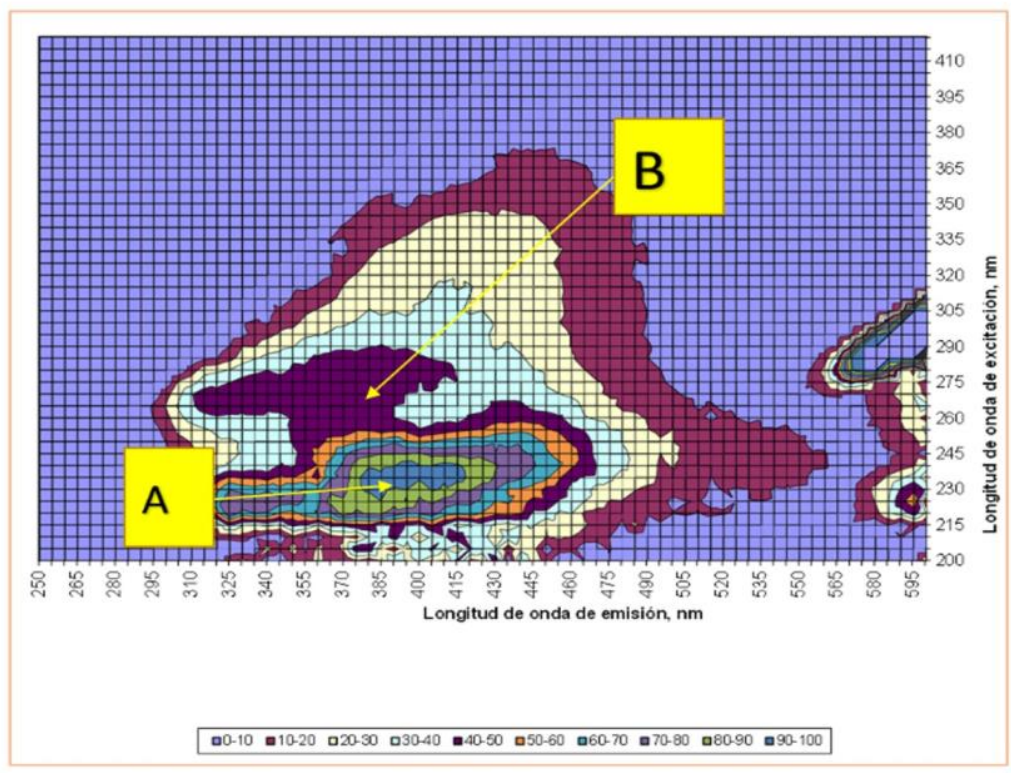

c)

Figura 10. EEM de DFC después del tratamiento de electro-oxidación: a) $10 \mathrm{mg} / \mathrm{l}$, b) $50 \mathrm{mg} / \mathrm{l}$ y c) $150 \mathrm{mg} / \mathrm{l}$. 


\section{Cinética de degradación del diclofenaco}

De acuerdo con la literatura, el diclofenaco presenta una cinética de degradación de primer orden (Brillas et al., 2010; Rivas, Gimeno, Borralho, \& Beltrán, 2010; Lu et al., 2017). La constante de velocidad es una constante de proporcionalidad directa entre la velocidad de reacción y la concentración de los reactivos; es decir, cuanto mayor es la concentración, mayor es la velocidad. El tiempo de vida media es el tiempo necesario para consumir la mitad de la concentración de un compuesto.

Se obtuvieron las constantes cinéticas y el tiempo de vida media del diclofenaco en cada uno de los experimentos, las cuales se muestran en la Tabla 5, observándose que en el experimento realizado con $\mathrm{NaCl}, 1.0$ A $\left(J=25.29 \mathrm{~A} / \mathrm{m}^{2}\right)$ y en ausencia de luz UV se presentó la constante de velocidad más alta $\left(1.08 \times 10^{-2} \mathrm{~min}^{-1}\right)$ y un tiempo de vida media de 63.89 min, la cual es similar a la obtenida por Brillas et al. (2010) de $1.02 \times$ $10^{-2}$ y $1.32 \times 10^{-2}$ a una intensidad de corriente de 300 y $450 \mathrm{~mA}$, respectivamente. Mientras que al aplicar una intensidad de corriente de $0.5 \mathrm{~A}\left(\mathrm{~J}=12.64 \mathrm{~A} / \mathrm{m}^{2}\right)$ se obtuvo una constante de velocidad más baja $\left(4.5 \times 10^{-3} \mathrm{~min}^{-1}\right)$ y mayor tiempo de vida media del fármaco (153.3 min). 
Tecnología y

Ciencias $₫$ Agua
2021, Instituto Mexicano de Tecnología del Agua

Open Access bajo la licencia CC BY-NC-SA 4.0

(https://creativecommons.org/licenses/by-nc-sa/4.0/)

Tabla 5. Modelo cinético de la degradación del diclofenaco.

\begin{tabular}{|c|c|c|c|c|c|}
\hline \multicolumn{2}{|c|}{ Muestra } & Ecuación & $\begin{array}{c}K \\
\left(\mathrm{~min}^{-1}\right)\end{array}$ & $R^{2}$ & $\begin{array}{c}t_{1 / 2} \\
(m i n)\end{array}$ \\
\hline \multirow{4}{*}{$\mathrm{NaCl}$} & $0.5 \mathrm{~A}$ & $\ln \left(\frac{C}{C_{0}}\right)=-0.0045 t+0.104$ & $4.5 \times 10^{-3}$ & 0.99 & 153.30 \\
\hline & $1.0 \mathrm{~A}$ & $\ln \left(\frac{C}{C}\right)=-0.0108 t+0.1738$ & $1.08 \times 10^{-2}$ & 0.99 & 63.89 \\
\hline & $\begin{array}{c}0.5 \\
\text { A/UV }\end{array}$ & $\ln \left(\frac{C}{C_{0}}\right)=-0.0046 t+0.1034$ & $4.6 \times 10^{-3}$ & 0.99 & 150.00 \\
\hline & $\begin{array}{l}1.0 \\
\text { A/UV }\end{array}$ & $\ln \left(\frac{C}{C_{0}}\right)=-0.0048 t+0.1446$ & $4.8 \times 10^{-3}$ & 0.94 & 143.75 \\
\hline \multirow{4}{*}{$\mathrm{Na}_{2} \mathrm{SO}_{4}$} & $0.5 \mathrm{~A}$ & $\ln \left(\frac{C}{C}\right)=-0.007 t+0.0087$ & $7 \times 10^{-3}$ & 0.98 & 98.57 \\
\hline & $1.0 \mathrm{~A}$ & $\ln \left(\frac{C}{C}\right)=-0.0046 t-0.163$ & $4.6 \times 10^{-3}$ & 0.94 & 150.00 \\
\hline & $\begin{array}{l}0.5 \\
\text { A/UV }\end{array}$ & $\ln \left(\frac{C}{C_{0}}\right)=-0.0095 t+0.0523$ & $9.5 \times 10^{-3}$ & 0.97 & 72.63 \\
\hline & $\begin{array}{l}1.0 \\
\text { A/UV }\end{array}$ & $\ln \left(\frac{C}{C_{0}}\right)=-0.0056 t-0.1961$ & $5.6 \times 10^{-3}$ & 0.93 & 123.21 \\
\hline
\end{tabular}

\section{Análisis estadístico}


Para conocer el efecto que tiene cada una de las variables de estudio se realizó el algoritmo de Yates (Material Suplementario I) y el análisis de varianza. De acuerdo con los resultados, el efecto con mayor significancia es el electrolito soporte, ya que cuenta con un valor de $\mathrm{P}$ muy pequeño. La interacción $A B$ (intensidad de corriente-luz UV) es significativa, teniendo un nivel aproximado de $19 \%$; por lo tanto, existe una ligera interacción entre la intensidad de corriente y la luz UV. La interacción entre los tres efectos también cuenta con un valor significativo de $10 \%$, lo cual indica de nueva cuenta que existe interacción entre la intensidad de corriente, radiación UV y electrolito soporte.

\section{Eficiencia de corriente instantánea}

La eficiencia de corriente es la relación de la corriente consumida por la degradación de un contaminante orgánico, mientras que la eficiencia de corriente instantánea (ECI) es la eficiencia actual en el momento dado de la electro-oxidación (Linares-Hernández et al., 2017). La ECI se calculó con la siguiente ecuación:

$E C I=\frac{\left(C_{i}-C_{f}\right)}{8 I \Delta t} F V$ 
En donde $C_{i}$ y $C_{f}$ son la concentración inicial y final de DFC a un $\Delta t$, ambas en $\mathrm{g} / \mathrm{l} ; F$, la constante de Faraday $(96487 \mathrm{C} / \mathrm{mol}) ; V$, el volumen en litros; I, la intensidad de corriente aplicada (1.0 A (J = 25.29 A/m²)), $\Delta t$, la diferencia de tiempo en segundos.

De acuerdo con la Figura 11 se observa que la mejor eficiencia de corriente instantánea para los experimentos de 150 y $50 \mathrm{mg} / \mathrm{l}$ ocurre de 120 a 180 minutos de reacción, mientras que a $10 \mathrm{mg} / \mathrm{l}$ sucede a 10 minutos. Lo anterior se debe a que en ese momento es donde se encuentra la mayor presencia de radicales $\bullet \mathrm{OH}$. Por otra parte, la disminución de la ECI puede explicarse debido al agotamiento de los radicales libres en las proximidades de la superficie del electrodo y a la disminución de la concentración del fármaco.

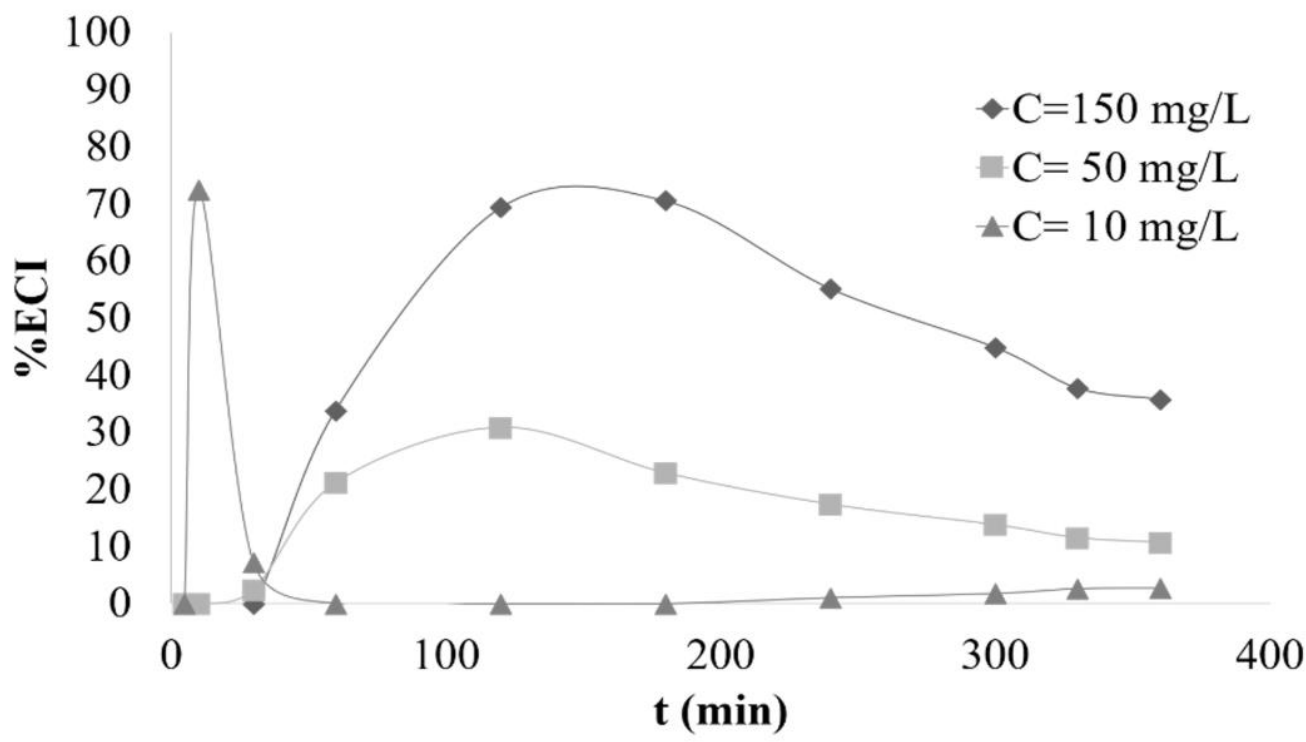

Figura 11. Porcentaje (\%) de ECI durante la reacción de electrooxidación de DCF. 


\section{Conclusiones}

El proceso de electro-oxidación con una configuración DDB-DDB resultó ser eficiente para la mineralización del diclofenaco; la degradación se logró debido a la generación de radicales hidroxilo, los cuales son unos de los oxidantes más fuertes.

El electrolito soporte es la variable que tiene más efecto dentro del proceso de oxidación, con un valor de $\mathrm{P}$ de 0.067 , y se determinó que el proceso se favorece al adicionar $\mathrm{NaCl}$ como electrolito soporte.

Las condiciones óptimas para una mejor eficiencia en la mineralización del diclofenaco fueron una intensidad de corriente de $1 \mathrm{~A}$ $\left(\mathrm{J}=25.29 \mathrm{~A} / \mathrm{m}^{2}\right)$; el valor de $\mathrm{pH}$ de la muestra $(5-6.4) ; \mathrm{NaCl}$ como electrolito soporte, en ausencia de luz UV, en un tiempo de 360 minutos, con una eficiencia de remoción de $97.8 \%$, mientras que COT, DQO Y $\mathrm{DBO}_{5}$ mostraron eficiencias de $64.4,89.3$ y $100 \%$, respectivamente.

La cinética de degradación del diclofenaco fue de pseudo primer orden, con un tiempo de vida media de 63.89 minutos.

\section{Agradecimientos}


Los autores agradecen el financiamiento otorgado por la UAEMEX, a través del proyecto No. 4482/2018/CI.

\section{Referencias}

Araujo, F. V. F., Yokoyama, L., Teixeira, L. A. C., Campos, J. C., Blanco, M., Martinez, A., Marcaide, A., Aranzabe, E., Aranzabe, A., Bonnineau, C., Moeller, A., Barata, C., Bonet, B., Proia, L., Sans-Piche, F., Guasch, H., Segner, H., Cherik, D., Benali, M., Louhab, K., Islam, M. S., Rouf, M. a, Kamaruddin, M. A., Yusoff, M. S., Ahmad, M. A., Le Thi Kim Phun and Le Anh Kien, ... Summary, E. (2011). Degradation Kinetics of Diclofenac in Water by Fenton ' s Oxidation. 2013, 16(4), 1-26. Recuperado de https://doi.org/10.1016/j.apcatb.2010.07.006

Archer, E., Petrie, B., Kasprzyk-hordern, B., \& Wolfaardt, G. M. (2017). Chemosphere The fate of pharmaceuticals and personal care products ( PPCPs ), endocrine disrupting contaminants ( EDCs ), metabolites and illicit drugs in a WWTW and environmental waters. Chemosphere, 174, 437-446. Recuperado de https://doi.org/10.1016/j.chemosphere.2017.01.101

Bae, S., Kim, D., \& Lee, W. (2013). Degradation of diclofenac by pyrite catalyzed Fenton oxidation. Applied Catalysis B: Environmental, 134135, 93-102. Recuperado de https://doi.org/10.1016/j.apcatb.2012.12.031

Barceló, L. D., \& López-de-Alda, M. J. (2008). Contaminación y calidad química del agua: el problema de los contaminantes emergentes. Fundación Nueva Cultura del Agua, 4(2), 125-128. Recuperado de 
https://doi.org/10.1097/ICB.0b013e3181ad3957

Barrera, C. (2014). Aplicaciones electroquímicas al tratamiento de aguas residuales. Barcelona, España: Reverté.

Bhadra, B. N., Seo, P. W., \& Jhung, S. H. (2016). Adsorption of diclofenac sodium from water using oxidized activated carbon. Chemical Engineering Journal, 301(April), 27-34. Recuperado de https://doi.org/10.1016/j.cej.2016.04.143

Bolong, N., Ismail, A. F., Salim, M. R., \& Matsuura, T. (2009). A review of the effects of emerging contaminants in wastewater and options for their removal. Desalination, 238(1-3), 229-246. Recuperado de https://doi.org/10.1016/j.desal.2008.03.020

Brillas, E., Garcia-Segura, S., Skoumal, M., \& Arias, C. (2010). Electrochemical incineration of diclofenac in neutral aqueous medium by anodic oxidation using Pt and boron-doped diamond anodes. Chemosphere, 79(6), 605-612. Recuperado de https://doi.org/10.1016/j.chemosphere.2010.03.004

Brillas, E., \& Sir??s, I. (2015). Electrochemical removal of pharmaceuticals from water streams: Reactivity elucidation by mass spectrometry. TrAC - Trends in Analytical Chemistry, 70, 112-121. Recuperado de https://doi.org/10.1016/j.trac.2015.01.013

Cao, D., Wang, Y., \& Zhao, X. (2017). Combination of photocatalytic and electrochemical degradation of organic pollutants from water. Current Opinion in Green and Sustainable Chemistry, 6, 78-84. Recuperado de https://doi.org/10.1016/j.cogsc.2017.05.007 
Casillas-García, J. E., Tzompantzi-Morales, F., Carbajal-Arizaga, G. G., López-Ganoa, A., \& Barrera-Rodríguez, A. (2017). Evaluación fotocatalítica de óxidos mixtos de Al203-Ga2O3 dopados con óxidos metálicos en la degradación de diclofenaco. Número Especial de La Revista Aristas: Investigación Básica y Aplicada, 6(11), 246-252. Recuperado de https://doi.org/ISSN 2007-9478

Casillas, J. E., Campa-Molina, J., Tzompantzi, F., Carbajal Arízaga, G. G., López-Gaona, A., Ulloa-Godinez, S., Cano, M. E., \& Barrera, A. (2017). Photocatalytic degradation of diclofenac using Al2O3-Nd2O3 binary oxides prepared by the sol-gel method. Materials, 13(6). Recuperado de https://doi.org/10.3390/ma13061345

Daughton, C. G., \& Brooks, B. W. (2011). Active pharmaceutical ingredients and aquatic organisms. Environmental Contaminants in Biota: Interpreting Tissue Concentrations, 286-347.

De-Franco, M. A. E., De Carvalho, C. B., Bonetto, M. M., De Pelegrini Soares, R., \& Féris, L. A. (2018). Diclofenac removal from water by adsorption using activated carbon in batch mode and fixed-bed column: Isotherms, thermodynamic study and breakthrough curves modeling. Journal of Cleaner Production, 181, 145-154. Recuperado de https://doi.org/10.1016/j.jclepro.2018.01.138

Fajardo, D., \& Córdoba, R. (2005). Plan de monitoreo para la planta de tratamiento de aguas residuales en el sur de Ahuachapán. UICNUnión Mundial Para La Naturaleza, 1, 1-24. Recuperado de https://cidoc.marn.gob.sv/documentos/plan-de-monitoreo-para-laplanta-de-tratamiento-de-aguas-residuales-en-el-sur-de- 
ahuachapan-el-salvador-c-a-caserio-puente-arce-municipio-de-sanfrancisco-menendez-departamento-de-ahuachapan/

Fatehifar, M., Borghei, S. M., \& Ekhlasi Nia, A. (2018). Application of moving bed biofilm reactor in the removal of pharmaceutical compounds (diclofenac and ibuprofen). Journal of Environmental Chemical Engineering, 6(4), 5530-5535. Recuperado de https://doi.org/10.1016/j.jece.2018.08.029

García-Montoya, M. F., Gutiérrez-Granados, S., Alatorre-Ordaz, A., Galindo, R., Ornelas, R., \& Peralta-Hernández, J. M. (2015). Application of electrochemical/BDD process for the treatment wastewater effluents containing pharmaceutical compounds. Journal of Industrial and Engineering Chemistry, 31, 238-243. Recuperado de https://doi.org/10.1016/j.jiec.2015.06.030

Gurung, K., Chaker, M., Shestakova, M., \& Sillanpää, M. (2018). Removal of carbamazepine from MBR effluent by electrochemical oxidation (EO) using a Ti / Ta2O5-SnO2 electrode Applied Catalysis B: Environmental Removal of carbamazepine from MBR e ffl uent by electrochemical oxidation ( EO ) using a Ti / Ta 205 -Sn. Applied Catalysis B: Environmental, 221(February), 329-338. Recuperado de https://doi.org/10.1016/j.apcatb.2017.09.017

Hurwitz, G., Pornwongthong, P., Mahendra, S., \& Hoek, E. M. V. (2014). Degradation of phenol by synergistic chlorine-enhanced photoassisted electrochemical oxidation. Chemical Engineering Journal, 240 , 235-243. Recuperado

de https://doi.org/10.1016/j.cej.2013.11.087 
Jaafarzadeh, N., Ghanbari, F., \& Moradi, M. (2015). Photo-electrooxidation assisted peroxymonosulfate for decolorization of acid brown 14 from aqueous solution, Korean Journal of Chemical Engineering, 32(3), 458-459. Recuperado de https://doi.org/10.1007/s11814014-0263-4

Jewell, K. S., Falås, P., Wick, A., Joss, A., \& Ternes, T. A. (2016). Transformation of diclofenac in hybrid biofilm-activated sludge processes. Water Research, 105, 559-567. Recuperado de https://doi.org/10.1016/j.watres.2016.08.002

Keen, O. S., Thurman, E. M., Ferrer, I., Dotson, A. D., \& Linden, K. G. (2013). Dimer formation during UV photolysis of diclofenac. Chemosphere, 93(9), 1948-1956. Recuperado de https://doi.org/10.1016/j.chemosphere.2013.06.079

Krajišnik, D., Daković, A., Malenović, A., Milojević-Rakić, M., Dondur, V., Radulović, Ž., \& Milić, J. (2013). Investigation of adsorption and release of diclofenac sodium by modified zeolites composites. Applied Clay Science, 83-84, 322-326. Recuperado de https://doi.org/10.1016/j.clay.2013.08.011

Linares Hernández, I., Barrera Díaz, C., Valdés Cerecero, M., Almazán Sánchez, P. T., Castañeda Juárez, M., \& Lugo Lugo, V. (2017). Soft drink wastewater treatment by electrocoagulation-electrooxidation processes. Environmental Technology (United Kingdom), 38(4), 433442.

Recuperado

de

https://doi.org/10.1080/09593330.2016.1196740

Liu, J., \& Wong, M. (2013). Pharmaceuticals and personal care products 
Tecnología y

Ciencias $₫$ Agua
2021, Instituto Mexicano de Tecnología del Agua

Open Access bajo la licencia CC BY-NC-SA 4.0

(https://creativecommons.org/licenses/by-nc-sa/4.0/)

(PPCPs): A review on environmental contamination in China. Environment International, 59, 208-224. Recuperado de https://doi.org/10.1016/j.envint.2013.06.012

Lonappan, L., Rouissi, T., Liu, Y., Brar, S. K., \& Surampalli, R. Y. (2019). Removal of diclofenac using microbiochar fixed-bed column bioreactor. Journal of Environmental Chemical Engineering, 7(1), 102894. Recuperado de https://doi.org/10.1016/j.jece.2019.102894

Lu, X., Shao, Y., Gao, N., Chen, J., Zhang, Y., Xiang, H., \& Guo, Y. (2017). Degradation of diclofenac by UV-activated persulfate process: Kinetic studies, degradation pathways and toxicity assessments. Ecotoxicology and Environmental Safety, 141(March), 139-147. Recuperado de https://doi.org/10.1016/j.ecoenv.2017.03.022

Martínez, C., Canle L., M., Fernández, M. I., Santaballa, J. A., \& Faria, J. (2011). Aqueous degradation of diclofenac by heterogeneous photocatalysis using nanostructured materials. Applied Catalysis B: Environmental, 107(1-2), 110-118. Recuperado de https://doi.org/10.1016/j.apcatb.2011.07.003

McGettigan, P., \& Henry, D. (2013). Use of Non-Steroidal AntiInflammatory Drugs That Elevate Cardiovascular Risk: An Examination of Sales and Essential Medicines Lists in Low-, Middle-, and High-Income Countries. PLoS Medicine. Recuperado de https://doi.org/10.1371/journal.pmed.1001388

Nieto-Sandoval, J., Munoz, M., de Pedro, Z. M., \& Casas, J. A. (2018). Fast degradation of diclofenac by catalytic hydrodechlorination. Chemosphere, 213, 141-148. Recuperado de 
https://doi.org/10.1016/j.chemosphere.2018.09.024

Pérez-Estrada, L. A., Malato, S., Gernjak, W., Agüera, A., Thurman, E. M., Ferrer, I., \& Fernández-Alba, A. R. (2005). Photo-fenton degradation of diclofenac: Identification of main intermediates and degradation pathway. Environmental Science and Technology, 39(21), 83008306. Recuperado de https://doi.org/10.1021/es050794n

Pourzamani, H., Hajizadeh, Y., \& Mengelizadeh, N. (2018). Application of three-dimensional electrofenton process using MWCNTsFe304nanocomposite for removal of diclofenac. Process Safety and Environmental Protection, 119, 271-284. Recuperado de https://doi.org/10.1016/j.psep.2018.08.014

Rivas, J., Gimeno, O., Borralho, T., \& Beltrán, F. (2010). Influence of oxygen and free radicals promoters on the UV-254nm photolysis of diclofenac. Chemical Engineering Journal, 163(1-2), 35-40. Recuperado de ttps://doi.org/10.1016/j.cej.2010.07.027

Rodríguez, E. M., Márquez, G., León, E. A., Álvarez, P. M., Amat, A. M., \& Beltrán, F. J. (2013). Mechanism considerations for photocatalytic oxidation, ozonation and photocatalytic ozonation of some pharmaceutical compounds in water. Journal of Environmental Management, 127, 114-124. Recuperado de https://doi.org/10.1016/j.jenvman.2013.04.024

Ross, A. B., \& Neta, P. (1982). Rate Constants for Reactions of Aliphatic Carbon-Centered Radicals in Aqueous Solution. National Bureau of Standards, National Standard Reference Data Series, 17(3). Recuperado de https://doi.org/10.1063/1.555978 
Sánchez-Montes, I., Pérez, J. F., Sáez, C., Rodrigo, M. A., Cañizares, P., \& Aquino, J. M. (2020). Assessing the performance of electrochemical oxidation using DSA $\AA$ and BDD anodes in the presence of UVC light. Chemosphere, $238 . \quad$ Recuperado de https://doi.org/10.1016/j.chemosphere.2019.124575

Tominaga, F. K., Dos Santos Batista, A. P., Silva Costa Teixeira, A. C., \& Borrely, S. I. (2018). Degradation of diclofenac by electron beam irradiaton: Toxicitiy removal, by-products identification and effect of another pharmaceutical compound. Journal of Environmental Chemical Engineering, 6(4), 4605-4611. Recuperado de https://doi.org/10.1016/j.jece.2018.06.065

Venegas-Orozco, M. J. (2013). Oxidación de anti-inflamatorios no esteroideos presentes en agua aplicando peróxido de Hidrógeno luz solar y nanopartículas de Hierro soportadas en zeolitas (tesis de maestría). Universidad Nacional Autónoma de México, México.

Vieno, N., \& Sillanpää, M. (2014). Fate of diclofenac in municipal wastewater treatment plant - A review. Environment International, 69 , 28-39. Recuperado de https://doi.org/10.1016/j.envint.2014.03.021

Yang, B., Wei, T., Xiao, K., Deng, J., Yu, G., Deng, S., Li, J., Zhu, C., Duan, H., \& Zhuo, Q. (2018). Effective mineralization of anti-epilepsy drug carbamazepine in aqueous solution by simultaneously electrogenerated $\mathrm{H} 2 \mathrm{O} 2 / \mathrm{O} 3$ process. Electrochimica Acta. Recuperado de https://doi.org/10.1016/j.electacta.2018.09.067

Žilnik, L. F., Jazbinšek, A., Hvala, A., Vrečer, F., \& Klamt, A. (2007). 
Solubility of sodium diclofenac in different solvents. Fluid Phase

Equilibria, 261(1-2), 140-145. Recuperado de https://doi.org/10.1016/j.fluid.2007.07.020 